\title{
Abstracts of the 5th International Conference of the Society for Heart and Vascular Metabolism
}

\author{
CD36 Mediates Fatty Acid Uptake and Triglyceride \\ Storage and Contributes to Dyslipidemia Associated \\ with Diet-induced Obesity
}

Debby P.Y. Koonen ${ }^{1}$, Rene L. Jacobs ${ }^{2}$, Maria Febbraio ${ }^{3}$, Martin E. Young ${ }^{4}$, Huy Ong ${ }^{5}$, Dennis E. Vance ${ }^{2}$, and Jason R.B. Dyck ${ }^{1}$

${ }^{1}$ Cardiovascular Research Group and the ${ }^{2}$ CIHR Group on the Molecular and Cell Biology of Lipids, Depts of ${ }^{1}$ Pediatrics and ${ }^{2}$ Biochemistry, Faculty fo Medicine and Dentistry, University of Alberta, Edmonton, Alberta, Canada. ${ }^{3}$ Department of Cell Biology, Lerner Research Institute, The Cleveland Clinic Foundation, Cleveland, Ohio, USA. ${ }^{4}$ Baylor College of Medicine, USDA/ARS Children's Nutrition Research Center, Houston, Texas, USA. ${ }^{5}$ Faculty of Pharmacy and Department of Pharmacology, Faculty of Medicine, Université de Montreal, Montreal, Quebec, Canada.

Consuming diets rich in fatty acids leading to excessive weight gain has been identified as a major contributor to the pathogenesis of Type 2 Diabetes (T2D), a known risk factor for cardiovascular disease. Associated with diet-induced obesity (DIO) are elevated levels of plasma fatty acids (FA) and lipoprotein associated triglycerides (TG). Since the levels of plasma FA and lipoprotein associated TG are primarily controlled by the liver, it is likely that aberrant hepatic FA uptake and handling contributes to the dyslipidemia that precedes development of T2D. Indeed, we have shown that mRNA and protein expression of the FA transport protein, CD36, which is normally expressed at low levels in the mouse liver, is significantly elevated during DIO and is correlated with increased hepatic TG storage and TG secretion as very low-density lipoproteins (VLDL). In vivo gene delivery of a recombinant adenovirus harboring CD36 cDNA (Ad.CD36) into lean mice showed a significant increase in hepatic FA uptake in vivo and an accumulation in hepatic TG storage and VLDL-TG secretion compared to Ad.Null-injected mice, demonstrating that increased CD36 expression alone is sufficient to recapitulate the alterations in liver lipid storage and VLDL-TG secretion observed in DIO. Together these data show that increased hepatic CD36 expression may play a causative role in the dyslipidemia associated with DIO and further suggests that inhibition of hepatic CD36 may prove to be beneficial for the prevention of insulin resistance and the eventual development of T2D.

OMEGA-3 Polyunsaturated Fatty Acid Incorporation Improves Oxygen Efficiency and Reduces Skeletal Muscle Fatigue in Rat

Gregory E. Peoples ${ }^{1}$, Peter L. McLennan ${ }^{2}$

School of Health Sciences ${ }^{l}$ and Graduates School of Medicine $^{2}$, University of Wollongong, Australia.

Background: Fish oil feeding increases incorporation of the omega-3 polyunsaturated fatty acid (n-3 PUFA) docosahexaenoic acid (DHA; 22:6n-3) into both skeletal muscle and myocardial cell membranes and increases the efficiency of myocardial oxygen use (1).

Objectives: To test the hypothesis that skeletal muscle oxygen consumption and fatigue may be influenced by $n-3$ PUFA.

Methods: Wistar rats were fed one of three diets (NuMega, High-DHA Tuna Fish Oil; n-3 PUFA, Safflower oil; n-6 PUFA, Saturated fat; SF) for $8 \mathrm{w}$. Oxygen consumption and muscle function were investigated using the in vivo, autoperfused, contracting hind limb with isometric tension development measured from the left gastrocnemius-plantarissoleus muscle bundle. Rats, stimulated via the sciatic nerve, were subjected to $3 \mathrm{x} 10 \mathrm{~min}(1 \mathrm{~Hz})$ stimulation bouts with $30 \mathrm{~min}$ rest between or a single bout $(2 \mathrm{~Hz})$ to $50 \%$ fatigue.

Results: Skeletal muscle of rats fed the n-3 PUFA, fatigued more slowly during stimulation compared to n-6 PUFA or SF, $p<0.05$ with less decrement in peak tension in successive stimulation bouts $p<0.05$. Muscle oxygen consumption was lowest in the n-3 PUFA group at rest and during stimulation increased by up to $413 \%$ (n-3PUFA) or $575 \%$ (SF) $(p<0.05)$. During fatiguing contractions, hind limb oxygen consumption relative to developed force was significantly less in n-3 PUFA rats.

Conclusions: Contracting skeletal muscle from fish oil fed rats has low oxygen consumption and is fatigue resistant compared to SF or n-6 PUFA feeding. This may reflect dietary 
induced changes in efficiency of oxygen use as seen in the heart.

(1) Pepe S, McLennan PL. Circulation 2002; 105: 2303-2308.

\section{Myocardial Fatty Acid Oxidation Rates Remain Elevated in $\mathrm{Ob} / \mathrm{Ob}$ Mice Despite Reversal of Obesity and Diabetes by Caloric Restriction}

\author{
Crystal Sloan, Joseph Tuinei, E. Dale Abel \\ University of Utah, Salt Lake City, UT, USA
}

Rationale: Leptin deficient ob/ob mice are commonly used to study the effects of obesity and insulin resistance on myocardial substrate metabolism. However, it is difficult to discern the specific effects of obesity/ insulin resistance/ diabetes versus leptin deficiency. We therefore adopted a strategy using caloric restriction to normalize body weight and reverse insulin resistance to study the effect of leptin deficiency on myocardial metabolism in lean ob/ob mice.

Methods: Male 4-week old ob/ob mice were "pair fed" to a leptin-treated group ( $3 \mathrm{mg} / \mathrm{kg} /$ day by i.p. injection) for three weeks. Glucose tolerance, serum insulin, serum triglycerides and FA oxidation rates in hearts perfused with $0.4 \mathrm{mM}$ palmitate $\pm 1 \mathrm{nM}$ insulin were determined.

Results: Leptin-treated ob/ob mice ate significantly less than non-treated ob/ob mice, and their weight returned to wildtype levels. Hyperglycemia, hyperinsulinemia, hypertriglyceridemia and cardiac hypertrophy were also completely reversed in leptin-treated ob/ob mice. In contrast, cardiac hypertrophy persisted in pair fed ob/ob mice and despite normalization of glucose tolerance and insulin levels, serum triglyceride concentrations increased by 2 and 6-fold in pair fed ob/ob mice relative to untreated ob/ob and wild-type mice, respectively $(p<0.05)$. As previously reported, palmitate oxidation rates were 2.1-fold increased in ob/ob hearts relative to controls $(p<0.005)$ and were normalized with leptin treatment. In contrast, palmitate oxidation rates remained elevated in pair fed ob/ob mice relative to wildtype controls (1.9-fold, $p<0.01)$. Insulin has a positive inotropic effect in perfused wildtype hearts, and this effect is absent in ob/ob hearts. Myocardial insulin sensitivity was restored by leptin treatment in ob/ob mice, but not in pair fed ob/ob mice despite equivalent body weights and glucose tolerance.

Conclusions: These data support a direct antihypertrophic effect of leptin in the heart and also suggest that leptin deficiency may directly contribute to myocardial insulin resistance and abnormal FA metabolism. Potential mechanisms include increased hepatic triglyceride production in leptin deficiency or direct effects of leptin signaling in the hypothalamus and/or the periphery on myocardial substrate utilization.
Hyperinsulinemic Normoglycemic Clamp Results in Hypolipidemia During Coronary Artery Surgery

Coert J. Zuurbier, Frans Hoek, Jasper van Dijk and Harry van Wezel.

Dept. of Anesthesiology and Clinical Chemistry, Academic Medical Center, Amsterdam

Background This study sought to quantify the effects of a perioperative hyperinsulinemic normoglycemic clamp on plasma levels of free fatty acids (FFA), triglyceride (TG) lactate and ketone bodies during coronary artery bypass grafting (CABG). Methods Forty-four nondiabetic patients with normal left ventricular function, scheduled for elective $\mathrm{CABG}$, were randomized to either a control group $(n=21)$ receiving standard care or to a clamp GIK group $(n=23)$ receiving a perioperative hyperinsulinemic $\left(0.1 \mathrm{IU} \mathrm{kg}^{-1} \mathrm{~h}^{-1}\right.$ insulin) normoglycemic (plasma glucose between 3.0 and $6.0 \mathrm{mmol} \mathrm{L}^{-1}$ ) clamp during $26 \mathrm{~h}$. We measured plasma insulin, glucose, FFA, lactate and ketone bodies at regular intervals from induction of anesthesia through the end of the second postoperative day (POD). Test tube lipolysis was prevented through the use of tetrahydrolipstatin (orlistat).

Results In the control group FFA peaked at $0.76 \pm 0.05 \mathrm{mM}$ at early reperfusion and decreased to $0.3-0.5 \mathrm{mM}$ during the remaining part of the protocol. GIK decreased FFA levels from $0.38 \pm 0.05 \mathrm{mM}$ at early reperfusion, to $0.10 \pm 0.01 \mathrm{mM}$ during the clamp. During the clamp period, GIK reduced the area under the curve for FFA by $75 \%$ and for TG by $53 \%$. GIK significantly increased lactate levels during early reperfusion from $1.2 \mathrm{mM}$ to $1.6 \mathrm{mM}$. Ketone bodies during the clamp period were significantly decreased, as reflected by a $50 \%$ smaller area under the curve in plasma ketone bodies.

Conclusions A hyperinsulinemic normoglycemic clamp during CABG decreases plasma FFA to very low levels of $<0.1 \mathrm{mM}$, with small increases in lactate and a $50 \%$ reduction in ketone bodies. The very low levels of FFA may diminish the potential benefits of GIK treatment.

Transgenic Suppression of Amp-Activated Protein Kinase Activity does not Impair Recovery of Cardiac Power During Reperfusion of Ischemic Hearts

Clifford D.L. Folmes ${ }^{1}$, Cory S. Wagg ${ }^{1}$, Mei Shen ${ }^{2}$, Rong Tian $^{2}$, Alexander S. Clanachan ${ }^{1}$, and Gary D. Lopaschuk ${ }^{1}$. ${ }^{1}$ Cardiovascular Research Group, University of Alberta and ${ }^{2}$ NMR Laboratory for Physiological Chemistry, Division of Cardiovascular Medicine, Brigham and Women's Hospital, Harvard Medical School

Activation of AMP-activated protein kinase (AMPK) has been suggested to benefit the heart during ischemia/ 
reperfusion by increasing energy production. While AMPK stimulates glycolysis, mitochondrial oxidative metabolism is the major source of cardiac ATP production during reperfusion of ischemic hearts. Stimulating AMPK increases mitochondrial fatty acid oxidation, but this is usually accompanied by a decrease in glucose oxidation. To examine the relationship between AMPK activation and cardiac energetics, we measured energy metabolism in hearts from mice expressing a dominant negative AMPK $\alpha 2$ (dnAMPK). Isolated working mouse hearts ( $n=4$ hearts/ group) were subjected to $30 \mathrm{~min}$ aerobic perfusion, $20 \mathrm{~min}$ global ischemia and $40 \mathrm{~min}$ of aerobic reperfusion with Krebs-Henseleit solution containing $5 \mathrm{mM}\left[\mathrm{U}-{ }^{14} \mathrm{C}\right]$ glucose, $0.4 \mathrm{mM}\left[9,10-{ }^{3} \mathrm{H}\right]$ palmitate and $100 \mu \mathrm{U} / \mathrm{ml}$ insulin. As expected, the dnAMPK hearts had reduced AMPK activity at the end of reperfusion $(82 \pm 9 \mathrm{vs} 141 \pm 7 \mathrm{pmol} / \mathrm{mg} / \mathrm{min}, P$ $<0.05$ ) with no changes in high-energy phosphates. Despite this, dnAMPK hearts had improved recovery of contractile function during reperfusion compared to wildtype controls $\left(15 \pm 0.8\right.$ vs $10 \pm 1.4$ bp $\left.\mathrm{mmHg} 10^{-3}, P<0.05\right)$. During the initial aerobic period, rates of glucose oxidation were higher in the dnAMPK hearts $(2060 \pm 158$ vs $1089 \pm 117$ $\mathrm{nmol} / \mathrm{g}$ dry $\mathrm{wt} / \mathrm{min}, P<0.05$ ) but palmitate oxidation was not different. Therefore total TCA cycle activity was also elevated $(5.2 \pm 0.3$ vs $3.2 \pm 0.5 \mu \mathrm{mol} / \mathrm{g}$ dry wt $/ \mathrm{min}, P<0.05)$, which is not accounted for with a $12 \%$ increase in function. These data demonstrates that suppression of cardiac AMPK activity does not produce an energetically compromised phenotype and does not impair, but may in fact improve, the recovery of mechanical function following ischemia.

\section{The AMPK-induced P70s6k Inhibition Participates in the Synergistic Effect of Biguanides and Insulin on Glucose Uptake Via the Overphosphorylation of the Pkb/As160 Axis}

Christophe Beauloye ${ }^{1}$, Audrey Ginion ${ }^{1}$, Céline Mouton ${ }^{1}$, Louis Hue $^{2}$, Jean-Louis Vanoverschelde ${ }^{1}$, Luc Bertrand ${ }^{1}$

${ }^{1}$ Division of Cardiology and ${ }^{2}$ Christian de Duve Institute of Cellular Pathology, Hormone and Metabolic research unit, Université catholique de Louvain, Brussels, Belgium.

Background: Biguanides, like metformin and phenformin, are known to lower blood glucose concentration by increasing muscle glucose disposal. Several findings suggest that the effects of biguanides could be mediated by the activation of AMP-activated protein kinase (AMPK). The aim of this work was to study the relationship between AMPK and the biguanide-stimulated glucose uptake in cardiomyocytes.

Methods: Cultured rat cardiomyocytes were incubated with insulin and phenformin, alone or in combination. Glucose uptake was measured by the detritriation rate of $\left[2-{ }^{3} \mathrm{H}\right]$ glucose. The phosphorylation state of the different signalling elements was measured by immunoblot.

Results: The stimulation of glucose uptake by biguanides correlated to AMPK phosphorylation, whereas that by insulin correlated with protein kinase B (PKB) phosphorylation. When added together, insulin and phenformin induced a concomitant increase in AMPK phosphorylation and PKB overactivation, resulting to a synergistic overstimulation of glucose uptake. This phenformin-induced PKB overactivation is accompanied by the inhibition of p70 ribosomal S6 kinase (p70S6K) and of the phosphorylation of insulin receptor substrate 1 (IRS-1) on serine, both presumed to be involved in the negative feedback inhibition of the insulin signalling. In all conditions, PKB and AMPK activation fully correlated to the phosphorylation of Akt-substrate 160 (AS160), known to participate in glucose uptake stimulation.

Conclusions: AMPK activators induced overstimulation of glucose uptake in insulin-treated cardiomyocytes via the inhibition of the p70S6K negative feedback loop. This is accompanied by the overphosphorylation of the PKB/AS160 axis. Our results suggest that AMPK activation could be a potential therapeutic approach to treat insulin resistance.

\section{Hyperthyroidism Increases Substrate Metabolism in Proportion to Increased Contractile Function and Hypertrophy in the Rat Heart}

Lisa C Heather ${ }^{\text {a }}$, Mark A Cole ${ }^{\mathrm{a}}$, Richard P Leoni ${ }^{\mathrm{a}}$, Rhys Evans $^{\mathrm{b}}$ and Kieran Clarke ${ }^{\mathrm{a}}$

${ }^{\mathrm{a} C a r d i a c}$ Metabolism Research Group, Department of Physiology, Anatomy and Genetics, University of Oxford, Oxford, UK. ${ }^{b}$ Nuffield Department of Anaesthetics, University of Oxford, Radcliffe Infirmary, Oxford, UK.

Objectives: Thyroid hormones have positive chronotropic and inotropic effects on the heart. We set out to investigate the effects of triidothyronine on cardiac substrate metabolism in the isolated, perfused, contracting heart.

Methods and Results: Triiodothyronine $(0.2 \mathrm{mg} \mathrm{kg}$ body weight $^{-1}$ day $^{-1}$ ip for 7 days) injected daily to male Wistar rats, increased heart weight by $34 \%$ and elevated plasma free fatty acids (FFA) by $97 \%$, compared with controls. Basal glycolytic rates per gram wet weight (gww) were the same for all hearts, but insulin-stimulated glycolysis was increased by $33 \%$ in hyperthyroid hearts. Palmitate oxidation and myocardial lipid incorporation rates were the same per gww for all hearts, with fatty acid metabolism increasing in proportion with the increased heart weight in the hyperthyroid hearts. There was a positive correlation between cardiac function and both fatty acid oxidation and glycolytic rates. Normalisation of metabolic rates to contractile function 
revealed no difference between hyperthyroid and control hearts. Protein levels of the fatty acid transporter, fatty acid translocase (FAT/CD36), were reduced by $46 \%$ in the hyperthyroid heart, and correlated negatively with plasma FFA concentrations. The protein levels of the other fatty acid transporters, plasma membrane and cytosolic fatty acid binding protein (FABPpm and cFABP) and fatty acid transport proteins (FATP) 1 and 6, were unchanged.

Conclusions: The hyperthyroid rat heart has increased substrate metabolism in proportion to increased contractile function and hypertrophy, resembling a physiological model of hypertrophy. The decreased FAT/CD36 protein levels may be to limit excessive fatty acid uptake in conditions in which circulating fatty acids levels are elevated.

\section{Enhancing cGMP Signaling in the Dystrophin-Deficient Heart Improves Contractile Function, Sarcolemmal Integrity and Mitochondrial Metabolism}

Maya Khairallah ${ }^{1}$, R. Khairallah ${ }^{1}$, M.E. Young ${ }^{2}$, C. Deschepper $^{3}$, B.G. Allen ${ }^{1}$, B.J. Petrof ${ }^{4}$, and C. Des Rosiers ${ }^{1}$ ${ }^{1}$ Montreal Heart Institute, ${ }^{2}$ Baylor College of Medicine, ${ }^{3}$ Institut de Recherches Cliniques de Montréal, and ${ }^{4}$ Meakins Christie Laboratories

Rationale. We recently found early metabolic alterations in the dystrophin-deficient $m d x$ heart that precede overt cardiomyopathy and may represent an early "sub-clinical" signature of a defective NO/cGMP pathway. Therefore, we hypothesize that early activation of this pathway, downstream of NO formation, will confer protection to $m d x$ hearts by enhancing their resistance to stress and reversing potentially maladaptive metabolic alterations.

Methods. We generated $m d x$ mice overexpressing in a cardiomyocyte-specific manner guanylate cyclase $(m d x / \mathrm{Tg})$ and assessed the metabolic and functional profile of these hearts using ex vivo perfusions in the working mode and a ${ }^{13} \mathrm{C}$-methodology.

Results. Compared to $m d x$ mice, $m d x / \operatorname{Tg}$ hearts from 12 weeks-old mice showed improved membrane integrity as evidenced by a $82 \pm 13 \%$ lower lactate dehydrogenase release while maintaining similar contractile function. In the presence of the transgene, the previously observed shift towards a preferential use of carbohydrates for energy production in the 12 weeks-old $m d x$ heart was emphasized, suggesting that this shift is adaptive. Nevertheless, myocardial citric acid cycle pool size was increased by $36 \pm 8 \%$ $(p<0.05)$, indicating improvement of previously documented mitochondrial metabolic alterations. With disease progression, at 5 months of age, membrane integrity was significantly improved by $63 \pm 12 \%$ and associated with a $179 \pm 30 \%$ increase in cardiac power $(p<0.05)$.

Conclusion. Enhancing cGMP signaling in the dystrophindeficient heart improved sarcolemmal integrity and contractile performance. These benefits were associated with reversal of potentially maladaptive mitochondrial metabolic alterations, and constitute a potential clinical therapeutic avenue for the dystrophic cardiomyopathy. (Funded by CIHR and HSFC)

\section{The AMPK Gamma 1 R70q Mutant Regulates Multiple Metabolic and Growth Pathways in the Cardiac Myocyte}

Karalyn D Folmes ${ }^{1}$, Lee A Witters ${ }^{2}$, Michael F Allard ${ }^{3}$, Martin E Young ${ }^{4}$ and Jason RB Dyck ${ }^{1}$.

${ }^{I}$ Cardiovascular Research Group, University of Alberta, Edmonton, AB, ${ }^{2}$ Dartmouth Medical School, Hanover, NH, ${ }^{3}$ James Hogg iCAPTURE Centre, University of British Columbia-St Paul's Hospital, Vancouver, BC, ${ }^{4}$ Children's Nutrition Research Center, Baylor College of Medicine, Houston, TX

Although mutations in the $\gamma$ subunit of AMP-activated protein kinase (AMPK) result in excessive glycogen accumulation and cardiac hypertrophy, the mechanisms by which this occurs have not been well defined. As greater than $65 \%$ of cardiac AMPK activity is associated with the $\gamma 1$ subunit of AMPK, we investigated the effects of expression of an AMPK-activating $\gamma 1$ subunit mutant ( $\gamma 1 \mathrm{R} 70 \mathrm{Q})$ on regulatory pathways controlling glycogen accumulation and cardiac hypertrophy in neonatal rat cardiac myocytes. While expression of $\gamma 1 \mathrm{R} 70 \mathrm{Q}$ displayed the expected increase in palmitate oxidation rates, rates of glycolysis were significantly depressed. Additionally, glycogen synthase activity was increased in cardiac myocytes expressing $\gamma 1 \mathrm{R} 70 \mathrm{Q}$, as a result of both increased expression and decreased phosphorylation of glycogen synthase. The inhibition of glycolysis and increased glycogen synthase activity were correlated with elevated glycogen levels in $\gamma 1 \mathrm{R} 70 \mathrm{Q}$-expressing myocytes. In association with the reduced phosphorylation of glycogen synthase, glycogen synthase kinase (GSK)-3 $\beta$ protein and mRNA levels were profoundly decreased in the $\gamma 1 \mathrm{R} 70 \mathrm{Q}-$ expressing myocytes. As GSK-3 $\beta$ is also a negative regulator of hypertrophy via inhibition of nuclear factor of activated $T$ cells (NFAT), the dramatic down-regulation of GSK-3 $\beta$ increased the nuclear activity of NFAT. Together, these data provide important new information as to the mechanisms by which a mutation in the $\gamma$ subunit of AMPK causes altered AMPK signaling and have identified multiple pathways involved in regulating both cardiac myocyte metabolism and growth that may contribute to the development of the $\gamma$ mutant-associated cardiomyopathy in humans. 
Dietary Supplementation With $\omega-3$ Polyunsaturated Fatty Acids Increases Plasma Adiponectin and Prevents Ventricular Remodeling and Dysfunction with Pressure Overload

W.C. Stanley, M.K. Duda, K.M. O'Shea, B. Lei, B.R. Barrows, A.M. Azimzadeh, T.E. McElfresh, B.D Hoit University of Maryland, Baltimore, and Case Western Reserve University, Cleveland, USA

Epidemiological studies suggest consumption of $\omega$ 3 -polyunsaturated fatty acids ( $\omega$-3-PUFA) decreases the risk for heart failure. In addition, recent studies show dietary supplementation with $\omega$-3-PUFA from fish increases the secretion of adiponectin, a cardioprotective adipokine. We hypothesized that supplementation with $\omega$-3-PUFA from fish oil increases plasma adiponectin and improves the adaptation of the left ventricle (LV) to arterial pressure overload. Rats underwent abdominal aortic banding or sham surgery $(n=9-$ 12 /group), and were fed standard chow or $\omega$-3-PUFA supplemented chow $(2.8 \%$ of energy intake as eicosapentaenoic acid + docosahexaenoic acid) for 8 weeks. Banding increased LV mass to a greater extent with the standard chow $(31 \%)$ than with $\omega$-3-PUFA $(18 \%)(p<0.05)$. With standard chow banding increased LV end diastolic and systolic volumes by $19 \%$ and $105 \%$, respectively, however they were unchanged with $\omega$-3-PUFA. Adiponectin mRNA expression in adipose tissue was increased $>2$-fold with $\omega$-3-PUFA, and plasma adiponectin concentration was elevated by $\sim 30 \%$ ( $p<$ $0.05)$, which correlated inversely with LV end systolic volume $(r=-0.46 ; p<0.05)$ in the banded animals. Supplementation with $\omega-3-P U F A$ did not affect expression of PPAR $\alpha$ regulated genes in the heart. Total protein expression of the pro-growth serine-threonine kinase Akt was increased two-fold by $\omega-3$ PUFA, but the fraction of Akt in the active phosphorylated form was decreased, and thus there were no differences in the amount of active P-Akt. The levels of total and phosphoAMPK were unaffected by either banding or diet.

In conclusion, dietary supplementation with $\omega$-3-PUFA prevented pressure overload induced LV dysfunction, which was associated with elevated plasma adiponectin.

\section{Resveratrol Activates Amp-activated Protein Kinase and Inhibits NFAT-Responsive Promoter Activity to Blunt Phenylephrine-Induced Cardiac Myocyte Hypertrophy}

Anita Y. M. Chan, Carrie-Lynn M. Soltys, and Jason R. B. Dyck

Cardiovascular Research Group, Departments of Pediatrics and Pharmacology, Faculty of Medicine, University of Alberta, Edmonton, Alberta, Canada T6G 2S2
Resveratrol, a polyphenol, has been attributed to some of the cardioprotective effects of red wine consumption, including the ability to inhibit hypertrophy via its antioxidant properties. Recently, resveratrol was found to activate AMP-activated protein kinase (AMPK) via indirect phosphorylation in hepatocytes. However, it is currently unknown whether resveratrol can also activate cardiac AMPK and the functional consequence of this. As we have shown that activation of AMPK inhibits protein synthesis associated with cardiac hypertrophy, we hypothesized that an additional mechanism by which resveratrol inhibits hypertrophy is via AMPK activation. To investigate this, neonatal rat cardiac myocytes were treated with vehicle or $50 \mu \mathrm{M}$ resveratrol for 24 hours and $10 \mu \mathrm{M}$ phenylephrine to induce hypertrophy. Activation of AMPK was achieved with resveratrol even in the presence of phenylephrine. $\left[{ }^{3} \mathrm{H}\right]$-phenylalanine incorporation revealed that resveratrol significantly decreased protein synthesis induced by phenylephrine. This was associated with reduced activities of regulators of protein synthesis, namely p70S6 kinase and eukaryotic elongation factor-2 (eEF2), as shown by phosphorylation changes on immunoblots. Specifically, resveratrol countered the effects of phenylephrine by blunting the increase in p70S6 kinase phosphorylation while partially restoring eEF2 phosphorylation. Furthermore, resveratrol dramatically decreased nuclear factor of activated T-cells (NFAT) promoter activity, as demonstrated by reduced luciferase activity driven by a NFAT-responsive luciferase reporter transgene. As the calcineurin-NFAT pathway is thought to be involved in pathological hypertrophy, this not only provides further evidence that AMPK activation has therapeutic potential, but also reveals another mechanism by which resveratrol can prevent hypertrophic growth.

\section{Substrate Oxidation Patterns and Insulin Sensitivity of the Isolated Working Rat Heart after Exercise Training or Chronic Pressure Overload}

Gracjan Pytel, Vitaly Maks, Michael Schwarzer, Torsten Doenst

University of Leipzig and Heart Center Leipzig, Germany

Hypothesis: We compared substrate oxidation patterns and the effect of insulin in isolated working rat hearts with physiological or pathological hypertrophy.

Methods: Physiological hypertrophy was induced by treadmill running of male Sprague Dawley rats for 10 weeks (TR). Pathological hypertrophy was induced by aortic banding for 10 weeks (AoB). Contractile function and hypertrophy grade was assessed by echocardiography. Glucose and fatty acid oxidation with or without insulin 
stimulation were assessed in the isolated working heart with radioactive tracers.

Results: Both interventions caused significant hypertrophy (LVPWD $+28 \%$ TR, $+68 \%$ AoB, both $p<0.01$ ). Ejection fraction was lowest after $\mathrm{AoB}(70 \pm 0.11 \%)$ and highest after TR $(82 \pm 0.06 \%)$ but the differences were not significant among groups. Isolated heart had lowest cardiac power after AoB (mW/gdry: $11.8 \pm 4.85$ vs. $40.8 \pm 1.65$ in Control, $p<0.01)$. TR did not affect cardiac power. Decreased cardiac power after AoB was associated with the lowest fatty acid oxidation rates ( $\mu$ molOleat/min/gdry AoB:0.29 0.06, TR:0.92 \pm 0.31 , Control:0.94 $\pm 0.25 p<0.01$ ). Glucose oxidation was decreased in both hypertrophy groups ( $\mu \mathrm{mol} / \mathrm{min} / \mathrm{gdry}:$ AoB:0.30 \pm 0.06 , TR:0.23 \pm 0.15 , Control: $0.53 \pm 0.12, p=0.014)$. Insulin stimulation increased glucose oxidation ( $\mu \mathrm{mol} \mathrm{min} \mathrm{m}^{-1}$ dry: AoB to $0.64 \pm 0.25$, TR: to $0.97 \pm 0.38$, Control: to $1.64 \pm 0.46)$. The relative increase was greatest in TR $(+480 \%)$ and smallest in AoB $(+105 \%)$. Insulin caused a reduction in fatty acid oxidation, which was similar in all groups (AoB:-50\%, TR:-52\%, Control:-50\%).

Conclusions: Physiological hypertrophy is associated with an unexpected decrease in basal glucose oxidation but an increased responsiveness towards insulin. Pathological hypertrophy demonstrates decreased substrate oxidation rates in the isolated heart associated with reduced function and insulin sensitivity.

\section{Metabolic Alterations Beyond Fatty Acid Oxidation in PPAR $\alpha$ Null Mice Hearts}

R Gélinas ${ }^{1}$, B Bouchard ${ }^{1}, \mathrm{~J}_{\text {McDuff }}{ }^{1}, \mathrm{G} \mathrm{Charron}^{1}$ and $\mathrm{C}$. Des Rosiers ${ }^{1}$.

${ }^{1}$ Departments of Nutrition, Montreal Heart Institute, University of Montreal, Quebec, Canada.

Background and Objective: While a shift from fatty acid (FA) to carbohydrates (CHO) is considered beneficial for the heart, it is unclear why subjects with FA $\beta$-oxidation (FAO) defects are prone to cardiac decompensation under stress conditions. In this study, we sought for alterations in myocardial $\mathrm{CHO}$ metabolism and its regulation using PPAR $\alpha$ null mice as our animal model of FAO defects.

Methods: ${ }^{13} \mathrm{C}$-methodology was used to assess substrate flux through energy yielding pathways in hearts perfused ex vivo at two workloads. The expression of selected metabolic genes was also assessed using real time PCR.

Results: Compared to control C57BL/6 mice, working hearts from $\operatorname{PPAR} \alpha$ null mice displayed an impaired capacity to withstand a raise in preload, as reflected by a $20 \%$ decline in aortic flow and cardiac efficiency. Beyond the expected shift from FA (four-fold down) to carbohydrate (CHO: 2-fold up) $(p<0.001)$ at both preloads, these hearts depicted a 44\% lower glycolytic flux rate when expressed relative to that of pyruvate decarboxylation at the higher preload $(p<0.05)$, suggesting a mismatch between cytosolic and mitochondrial $\mathrm{CHO}$ metabolism. One potential mechanism for this mismatch is suggested by a $68 \%$ decrease in the gene expression of ATP-citrate lyase, an enzyme that may determine cytosolic levels of citrate, an inhibitor of glycolytic phosphofructokinase, in PPAR $\alpha$ null mice hearts $(p<0.05)$.

Conclusion: Collectively, our data highlight metabolic alterations in PPAR $\alpha$ null mice hearts, beyond their lower FAO, which may be determinant for their response to an increase in workload. (Supported by CIHR and NIH).

\section{High-Fat Feeding Increases Fatty Acid Oxidation Rates and Uncouples Respiration in Cardiac Mitochondria}

Andrew J Murray, Sara McAleese, Lindsay M Edwards and Kieran Clarke

University of Oxford, Department of Physiology, Anatomy and Genetics, Parks Road, Oxford, UK, OXI 3PT

High-fat feeding in rats decreases cardiac efficiency (hydraulic work $/ \mathrm{O}_{2}$ consumption) and impairs exercise capacity. The aim of this study was to determine whether high-fat feeding alters mitochondrial respiration in the heart by increasing uncoupling protein 3 (UCP3) levels. Male Wistar rats were fed standard chow $(n=6)$ or high-fat diet $(55 \%$ saturated fat) $(n=6)$ for one month. Hearts were excised and interfibrillar and subsarcolemmal mitochondria isolated. State III and state IV respiration rates were measured using an oxygen electrode, with glutamate or palmitoyl-carnitine as substrates, and $\mathrm{ADP} / \mathrm{O}$ ratios were calculated. Interfibrillar mitochondria from high-fat fed rats had higher state III respiration rates with palmitoyl-carnitine than those from chow-fed animals, and had lower ADP/O ratios, indicating uncoupling of oxidation/phosphorylation with both substrates. ADP/O ratios were the same in both groups in the presence of GDP, a UCP3 inhibitor (see Figure). Subsarcolemmal mitochondria from high-fat fed rats had higher state III respiration rates with palmitoyl-carnitine than those from chow-fed rats, and again had lower ADP/O ratios with both substrates. As with interfibrillar mitochondria, ADP/O ratios were the same in both groups in the presence of GDP. Cardiac UCP3 and mitochondrial thioesterase-1 protein levels, measured using immunoblotting, were both $20 \%$ higher in high-fat compared with chow-fed rats, with no difference in levels of the mitochondrial respiratory chain complexes. In conclusion, high-fat feeding increased mitochondrial fatty acid oxidation and uncoupled respiration via elevated UCP3 levels, probably through PPAR $\alpha$ activation. 


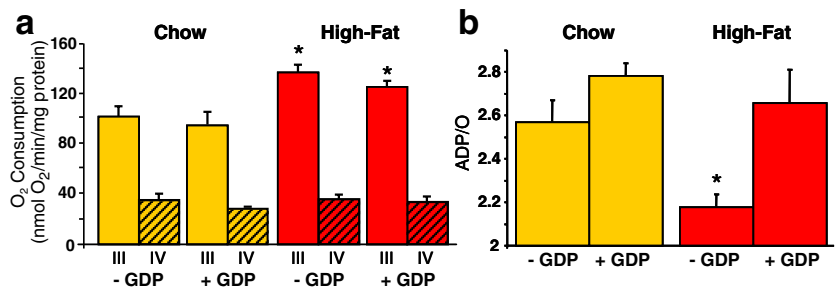

Figure a) State III and state IV respiration rates, and b) ADP/O ratios in interfibrillar mitochondria isolated from chow and high-fat fed rat hearts with palmitoyl-carnitine as substrate, and in the presence and absence of GDP, a UCP3 inhibitor.

${ }^{*} P<0.05$ compared with same parameter in interfibrillar mitochondria from chow-fed rat hearts.

UCP 3 Does Not Regulate Fatty Acid Oxidation Rates in the Heart but Controls Cardiac and Skeletal Muscle ROS Production

Sihem Boudina, Joseph Tuinei, Heather Theobald, Sandra Sena and E. Dale Abel

Division of Endocrinology, Metabolism and Diabetes and Program in Human Molecular Biology and Genetics, University of Utah School of Medicine, Salt Lake City, Utah 84112.

The biological functions of uncoupling protein 3 (UCP3) in cardiac muscle are incompletely understood and its role in maintaining coupling of oxidative phosphorylation in skeletal muscle is controversial. These studies were designed to investigate the role of UCP3 in cardiac and skeletal muscle energy metabolism under normal and high fat diets. Mice lacking UCP 3 (UCP3KO) and wildtype (WT) littermates were fed a normal chow (NC) or a high fat (HF) diet for 10 weeks. Palmitate oxidation (POX), myocardial oxygen consumption $\left(\mathrm{MVO}_{2}\right)$, cardiac efficiency (CE) and cardiac power were determined in isolated working hearts. Mitochondrial respiration and ATP synthesis were determined in saponin-permeabilized soleus and cardiac muscle. Oxidative damage was determined using dichlofluorescein (DCF). On NC, POX rates were similar between UCP3KO and WT but increased similarly with HF $(726.5 \pm 41.8$ vs. $731.7 \pm 47.1 \mathrm{nmol} / \mathrm{min} / \mathrm{mg} \mathrm{DHW}) . \mathrm{MVO}_{2}$, which increased in WT with HF $(P<0.05)$ did not increase in UCP3KO on a HF. CE and cardiac power were reduced in WT with HF but preserved in UCP3KO on HF. State 2, 3 and 4 were not different in cardiac and soleus fibers from WT and UCP3KO on NC. Compared to NC, state 4 respiration increased with $\mathrm{HF}$ by $26 \%$ and $34 \%$ in cardiac mitochondria from WT and UCP3KO respectively. On HF, ATP synthesis increased by $19 \%$ and $28 \%(P<0.05)$ in soleus and cardiac mitochondria from UCP3KO. Reactive oxygen species (ROS) generation was $49 \%(P<0.005)$ higher in hearts from WT on HF compared to WT on NC. However, ROS generation was further increased in UCP3KO on HF $(212.1 \pm 16.2$ versus $177.8 \pm 9.1, P<0.05)$. In skeletal muscle, ROS generation was not increased in WT on the HF diet but rose by $18 \%(P<0.005)$ in UCP3KO on HF compared to UCP3KO on NC. In conclusion, UCP3 mediates the increase in $\mathrm{MVO}_{2}$ that accompanies $\mathrm{HF}$ feeding and contributes to reducing fat-induced ROS accumulation. Despite these effects, mitochondrial ATP generation increased, indicating that UCP3 also regulates the efficiency of mitochondrial ATP generation.

Dietary Fish-Oil Preserves Cardiac Contractile Function by Upregulation of Ucp3 in a Rat Model of High-Fat Diet-induced Glucose Intolerance and Cardiomyopathy

D.M. Ouwens ${ }^{1}$, C.E. van den Brom $^{2}$, J. Kriek ${ }^{1}$, G. Schaart ${ }^{3}$, M.K. Hesselink ${ }^{3}$, P. Schrauwen ${ }^{3}$, R. Vlasblom ${ }^{2}$, M. Diamant ${ }^{4}$ ${ }^{1}$ Department of Molecular Cell Biology, LUMC, Leiden, ${ }^{2}$ Laboratory for Physiology, VUMC, Amsterdam, ${ }^{3}$ Department of Human Biology, Maastricht University, Maastricht, ${ }^{4}$ Department of Endocrinology, Diabetes Centre, VUMC, Amsterdam, The Netherlands

Background and Aims: Myocardial insulin resistance and lipotoxicity, leading to mitochondrial dysfunction and oxidative stress, have been implicated in the development of diabetic cardiomyopathy (DCM), a common complication in type 2 diabetes mellitus. Uncoupling protein 3 (UCP3) has been proposed to protect against lipid-induced mitochondrial damage, either by exporting excessive fatty acid anions from the mitochondrial matrix and/or by reducing mitochondrial ROS production and lipid peroxide formation, thus being an important protective mechanism in diabetes-related organ damage. We studied whether diet-induced alterations in myocardial insulin sensitivity and in vivo cardiac function in rats could be ascribed to changes in UCP3 protein expression. Materials and Methods: Male Wistar rats, fed isocaloric low-fat- (LF), high-fat- (HF), or high-fat fish-oil-enriched (FISH) diets, underwent an oral glucose tolerance test (oGTT) at day 25, and in vivo echocardiography at day 27. Rats were sacrificed at day 28 for analysis of myocardial protein expression and phosphorylation.

Results: No significant differences in body weight gain, fasting plasma glucose and insulin levels were found among the diet-groups. However, compared to LF-diet fed rats, HF- and FISH-diet fed rats showed glucose intolerance in the oGTT $(P<0.05)$. Also insulin-mediated activation of the $\mathrm{PKB} /$ Akt-pathway was $40-50 \%$ lower in hearts from both HF- and FISH-diet fed rats, compared to LF-diet fed 
hearts $(P<0.05)$. Interestingly, cardiac contractile function, expressed as fractional shortening and ejection fraction, was similar in FISH- and LF-diet fed rats, but was decreased in HF-diet fed rats $(P<0.05$ versus FISH). Myocardial protein levels of UCP3 were 1.9-fold increased in FISH-diet fed rats compared to LF-diet fed rats $(P<0.01)$, but failed to rise significantly in HF-diet fed rats.

Conclusion: FISH-oil enriched diet induced myocardial UCP3 expression while preserving cardiac contractile function in rats. We propose that dietary FISH-oil prevents lipotoxic heart disease by upregulation of UCP3, rather than by improving myocardial insulin signalling, in a rat model of diet-induced glucose intolerance.

\section{High Fat Fed Heart Failure Animals have Enhanced Mitochondrial Function and Acyl-CoA Dehydrogenase Activities}

Julie H Rennison ${ }^{1}$, Martin E Young ${ }^{2}$, Charles L Hoppel ${ }^{1}$, Margaret P Chandler ${ }^{1}$

Case Western Reserve University, Cleveland, OH; Baylor College of Medicine, Houston, TX' ${ }^{2}$ USA

We have previously shown that administration of high fat in heart failure (HF) increased mitochondrial respiration and did not alter left ventricular (LV) function. PPAR $\alpha$ is a nuclear transcription factor that activates expression of genes involved in fatty acid uptake and utilization. We hypothesized that elevated mitochondrial respiration in high fat fed $\mathrm{HF}$ rats is due to increased expression of $\beta$-oxidation enzymes (short (SCAD), medium (MCAD), and long (LCAD) chain acyl-CoA dehydrogenase). Rats underwent ligation or sham surgery and were fed normal (10\% kcal fat) (SHAM, HF) or high fat diet $(60 \%$ kcal saturated fat) (SHAM+FAT, HF+FAT) for 8 weeks. Subsarcolemmal and interfibrillar mitochondria were isolated from the LV. State 3 respiration using fatty acid substrates octanoylcarnitine and palmitoylcarnitine was increased in HF+FAT compared to SHAM+FAT and HF respectively $(242 \pm 21,246 \pm 21$ vs 183 $\pm 8,181 \pm 6$ and $193 \pm 17,185 \pm 16 \mathrm{nAO} / \mathrm{min} / \mathrm{mg}$ ). Despite decreased mcad mRNA in HF and HF+FAT, MCAD protein was not different between groups, and MCAD activity increased in HF+FAT $(65.1 \pm 2.7$ vs $81.5 \pm 5.4 \mathrm{nmol} / \mathrm{min} / \mathrm{mg})$. Activities of SCAD and LCAD were also elevated in HF+FAT though scad and lcad mRNA expression were not different. SCAD, MCAD, and LCAD activities correlated to increased state 3 respiration using both fatty acid substrates. In conclusion, enhanced mitochondrial respiration associated with high fat may result from increased activation of acylCoA dehydrogenases, but is not due to increased mRNA or protein expression. High fat in normal animals did not adversely affect mitochondrial respiration or the expression and activity of enzymes involved in $\beta$-oxidation.

\section{Regulation of Cardiac Substrate Utilization by Protein O-Glycosylation}

Arzu Onay-Besikci ${ }^{1}$, Norbert Fülöp ${ }^{2}$, Richard B Marchase ${ }^{2}$, John C Chatham ${ }^{2}$

${ }^{1}$ Faculty of Pharmacy, Ankara University, Turkey; ${ }^{2}$ Univ. Alabama, Birmingham, USA

Increased hexosamine biosynthesis (HB) flux and $O$-linked attachment of N-acetyl-glucosamine $(O-G l c N A c)$ on nucleocytoplasmic proteins have both been implicated in diabetic cardiomyopathy. The goal of this study was to determine the impact of acute increases in $\mathrm{HB}$ flux and $O$-GlcNAc levels induced by glucosamine $(\mathrm{GlcN})$ on cardiac function and substrate metabolism. Metabolic fluxes were determined in isolated hearts perfused with ${ }^{13} \mathrm{C}$-labeled substrates and $0,0.05,0.1,1,5$ and $10 \mathrm{mM}$ GlcN using ${ }^{13} \mathrm{C}-\mathrm{NMR}$ isotopomer analysis. UDP-GlcNAc, a product of $\mathrm{HB}$ and a precursor of $O$-GlcNAc was assessed by HPLC and protein $O$-GlcNAc levels were assessed by immunoblot analysis. GlcN had no effect on cardiac function at any concentration; however, both UDP-GlcNAc and $O$-GlcNAc were significantly increased with GlcN treatment with a maximal response at $1 \mathrm{mM}$ GlcN ( 2-fold increase vs $0 \mathrm{mM} ; p<0.05)$. The addition of $0.05 \mathrm{mM}$ GlcN significantly increased the contribution of palmitate $(47 \pm 3 \%$ vs $56 \pm 1 \%, p<0.05)$, and decreased the contribution of lactate $(28 \pm 3 \%$ vs $19 \pm 1 \%, p<0.05)$ to oxidative metabolism. Increasing GlcN to $0.1 \mathrm{mM}$ further increased palmitate oxidation and reduced lactate oxidation; however, higher concentrations had no additional effect on substrate utilization. Thus, GlcN significantly increased $O$-GlcNAc levels and altered fatty acid and carbohydrate oxidation in a similar manner to that seen with diabetes. These data suggest that changes in $O$-GlcNAc levels may contribute to the shifts in cardiac substrate utilization seen in diabetes. Supported in part by TUBİTAK 2219 (AOB); NIH HL067464, HL079364 (JCC); NIH HL076175 (RBM).

\section{CARDIAC Lipid Accumulation and Cardiac Function in Well-Controlled Type 2 Diabetes Mellitus Patients}

V.B. Schrauwen-Hinderling*\#, M.E. Kooi ${ }^{\S \#}$, M.K.C. Hesselink $^{*}$, R. Meex ${ }^{*}$, E. Phielix ${ }^{*}$, J.F.C. Glatz ${ }^{\S}$, P. Schrauwen ${ }^{*}$ ${ }^{*}$ NUTRIM, Maastricht University, ${ }^{\#}$ Department of Radiology, University Hospital Maastricht, ${ }^{\S}$ CARIM, Maastricht University, Maastricht 
Background: In cardiomyocytes, lipids and lipid metabolites can act as cardiotoxins, impairing cardiac function and increasing the risk for dilated cardiomyopathy. As the prevalence of cardiomyopathy is high in type 2 diabetes mellitus (T2DM), an increased deposition of intra-cardiocellular lipids (ICCL) may be expected.

Objective: To develop, validate and apply a non-invasive method to quantify ICCL in subjects with T2DM and BMImatched controls and to relate outcomes to recognized clinical markers of cardiac function.

Methods: So far, 6 patients with well-controlled T2DM and 12 controls were metabolically phenotyped and included in the study (age(y): $60.2 \pm 0.5$ vs $61.8 \pm 2.7$, BMI $\left(\mathrm{kg} / \mathrm{m}^{2}\right): 29.9 \pm 1.0$ vs $29.0 \pm 0.7$, T2DM vs controls, all males). Magnetic resonance imaging was used to investigate the hemodynamic cardiac function, and proton magnetic resonance spectroscopy to quantify ICCL in the intraventricular septum of the heart. Cardiac triggering and breathing commands were used to compensate for cardiac and respiratory motion. Measurements were performed on a 1.5T whole-body system (Intera, Philips Medical Systems). Results: The correlation of repeated ICCL-measurements was good $(R=0.96 ; p<0.05))$. The results show that ICCL is higher in T2DM, but the difference did not reach statistical significance $(15.4 \pm 4.1$ vs $10.8 \pm 1.5 \mathrm{mmol} / \mathrm{g}, p=$ $0.216)$. Interestingly, the variation between subjects is large in T2DM, suggesting that subtypes of diabetes may be more affected. ICCL content tended to correlate with the concentricity index (leftventricular mass/leftventricular volume, $n=14, R=0.528, p=0.05)$.

Conclusions: Here we present a reproducible, non-invasive method to quantify ICCL in humans. Inclusion of additional subjects is needed to make a definitive statement on ICCL content in T2DM. The preliminary data further indicate that an increased cardiac lipid content is accompanied by a concentric remodeling of the heart.

This work was made possible by a grant from the EFSD/ Lilly Research Fund. VB S-H was supported by a Kootstra Fellowship.

\section{Low Threshold Intake of Fish Oil for Myocardial Omega-3 Fatty Acid Incorporation is Not Subject to Competition with Omega-6 Fatty Acids}

\footnotetext{
${ }^{1}$ Peter L McLennan, ${ }^{2}$ Emily L Slee, ${ }^{2}$ Mandy L Theiss, ${ }^{3}$ Alice J Owen.

${ }^{1}$ Graduate School of Medicine, ${ }^{2}$ School of Health Sciences, University of Wollongong, NSW; ${ }^{3}$ Department of Epidemiology and Preventative Medicine, Monash University, Vic. Australia.
}

Background - Regular intake of fish or fish oil is associated with reduced risk of cardiovascular disease. Animal studies provide biologically plausible bases for omega-3 polyunsaturated fatty acids (PUFA) associated with incorporation into myocardial membrane phospholipid; however a link between regular low intakes of fish oil and membrane change has not been demonstrated, especially in the context of high omega-6 PUFA background diet.

Objective - To establish the dose-response of very low dietary FO intake in the rat and the influence of dietary omega-6 PUFA on heart omega-3 PUFA incorporation.

Methods - Rats were fed diets containing $0.12 \%$ en $-8.7 \%$ en as EPA+DHA from FO compared to $0 \% \mathrm{FO}$, with background diets containing either $1.5 \%$ en or $7.5 \%$ en as omega-6 PUFA (linoleic acid) for $4 \mathrm{w}$.

Results - Fish oil increased heart omega-3 content (mainly DHA) by 1.7 to 3.3 fold (from $7 \%$ (at $0 \% \mathrm{FO}$ ) to $25 \%$ membrane fatty acids) in a log-linear fashion with little EPA incorporation. A dietary threshold for membrane incorporation was established at $0.02 \%$ en EPA+DHA. High dietary n-6 PUFA had no significant effect on the slope or threshold for heart DHA incorporation.

Conclusions- Heart cell membranes are sensitive to the absolute amounts of long-chain omega-3 PUFA in the diet but not to the dietary ratio of omega-6/omega-3 PUFA, even at very low fish oil intakes. Doubling of rat myocardial membrane omega-3 PUFA composition was achieved with \%energy intakes equivalent to human intake of 1-2 meals of fatty fish per week.

\section{Nutritional Preconditioning of the Rat Heart by Dietary Fish Oil}

Peter L. McLennan ${ }^{1}$, Grace G. Abdukeyum ${ }^{2}$

${ }^{1}$ Graduate School of Medicine, ${ }^{2}$ School of Health Sciences, University of Wollongong, NSW 2522, Australia

Background: Dietary fish oil provides a range of cardioprotective effects associated with myocardial incorporation of long-chain omega-3 polyunsaturated fatty acids (n-3 PUFA). They prevent arrhythmias, lower heart rate and reduce oxygen consumption without detriment to work output.

Objectives: To compare the effects of dietary fish oil with those of ischaemic preconditioning during ischemia/ reperfusion.

Methods: Rats were fed diets with fish oil (n-3 PUFA), sunflower seed oil (n-6 PUFA) or saturated fat (beef tallow) (SF) for 6 weeks. Isovolumic, isolated perfused hearts were reperfused for $120 \mathrm{~min}$ after $30 \mathrm{~min}$ regional ischemia, with 
or without ischaemic preconditioning ( $3 \mathrm{x} 5 \mathrm{~min}$ cycles of global ischemia).

Results: Ischemic preconditioning significantly reduced heart rate, coronary flow and end-diastolic pressure (EDP) at rest, reduced EDP rise in ischaemia/reperfusion, increased developed pressure recovery and reduced arrhythmias post-ischaemia, but only in SF and n-6 PUFA hearts. The n-3 PUFA hearts had lower coronary flow, heart rate and EDP at rest. Post-ischaemic arrhythmias, heart rate, coronary flow and EDP rise were reduced, and recovery of developed pressure and maximum rate of relaxation were increased.

Conclusions: Prefeeding with n-3 PUFA provided cardioprotection, improving recovery, reducing stunning and arrhythmia while ischemic preconditioning provided similar but no additional protection in n-3 PUFA hearts. We conclude that dietary fish oil produces a preconditioninglike effect with chronic administration, which by virtue of the continuous presence of n-3 PUFA in the membrane is apparently not subject to desensitisation.

\section{Omega-3 Fatty Acids in Human Heart: Correlation Between Atria, Ventricle and Erythrocytes}

Mandy L Theiss ${ }^{1}$, Peter L McLennan ${ }^{1}$, Salvatore Pepe ${ }^{2}$, Freya Sheeran ${ }^{2}$

${ }^{1}$ University of Wollongong, ${ }^{2}$ Monash University, Australia

Background: Omega-3 polyunsaturated fatty acids (PUFA) are cardioprotective in animals. Human red blood cell (RBC) eicosapentaenoic acid and docosahexaenoic acid (EPA+DHA) correlates inversely with adverse cardiovascular outcomes and is proposed as a cardiovascular risk factor (Omega-3 Index) (Harris \& von Schacky, 2004) on the premise that it reflects the composition of the myocardium. However, animal tissues vary in omega-3 content and balance.

Objectives: To establish the relationships between EPA + DHA in human RBC, atria and ventricle.

Methods: This study evaluated the fatty acid composition of right atria and left ventricles from fresh donor hearts available through a heart transplant program $(n=20)$ and from right atrial biopsy and red blood cells, obtained during cardiac surgery $(n=10)$.

Results: DHA was the major omega-3 fatty acid in all tissues. Patterns of incorporation were different in different tissue types. No differences were seen between biopsy samples of right atria and donor right atria. EPA+DHA was highly correlated between RBC and right atria biopsy $\left(r^{2}=\right.$ $0.6, P=0.0001)$ and between right atria donor and left ventricle donor $\left(r^{2}=0.7, P<0.0001\right)$.

\begin{tabular}{lrrr}
\hline & \multicolumn{1}{l}{ EPA } & \multicolumn{1}{l}{ DHA } & EPA+DHA \\
\hline RBC & $* 1.15 \pm 0.58$ & $5.59 \pm 1.42$ & $* 6.74 \pm 1.69$ \\
Right atria biopsy & $0.58 \pm 0.71$ & $5.12 \pm 2.11$ & $5.71 \pm 2.68$ \\
Right atria donor & $0.75 \pm 0.35$ & $5.93 \pm 2.11$ & $6.70 \pm 2.40$ \\
Left ventricle donor & $0.77 \pm 0.38$ & $* * 4.51 \pm 1.22$ & $* * 5.28 \pm 1.55$
\end{tabular}

Data are mean \pm SD. ${ }^{*} P<0.05 \mathrm{v}$ right atria biopsy; ${ }^{*} P<0.0001 \mathrm{v}$ right atria donor; paired t-test.

Conclusions: Having established there was no difference in the levels of omega-3 EPA+DHA in right atrial samples between study groups, we conclude that EPA+DHA in human red blood cells is a valid marker of ventricular EPA+DHA.

\section{TTA is a Pan PPAR Ligand which Markedly Stimulates} Fatty Acid Oxidation Following In Vivo Administration

Ahmed M. Khalid ${ }^{1}$, Ellen Aasum ${ }^{1}$, Anne D. Hafstad ${ }^{1}$, Kieran Clarke ${ }^{3}$, Rolf Berge ${ }^{2}$, and Terje S. Larsen ${ }^{1}$

${ }^{1}$ Institute of Medical Biology, University of Tromsø, and

${ }^{2}$ Institute of Medicine, University of Bergen, Norway, Department of Physiology, Anatomy and Genetics, University of Oxford, UK

Peroxisome proliferator-activated receptor a (PPARa) is known to be important in long term regulation of fatty acid (FA) oxidation in the heart. Most studies, however, have shown little effect of PPAR $\alpha$ agonists on myocardial PPAR target genes or cardiac FA oxidation when administered in vivo. In the present study we examined metabolic effects of tetradecylthioacetic acid (TTA, a pan PPAR agonist) on hearts from Balb/c mice. The animals were treated for 8 days (TTA $0.5 \%$, added to the diet), after which myocardial FA and glucose oxidation, myocardial oxygen consumption $\left(\mathrm{MVO}_{2}\right)$ and cardiac work (pressure-volume area, PVA) were measured using the isolated working heart model. Changes in cardiac gene expression were measured with RT-PCR.

TTA treatment resulted in a near 2.5 fold increase in myocardial FA oxidation with a concomitant $-47 \%$ reduction in glucose oxidation. This change in cardiac metabolism occurred in the face of a significant reduction in circulating plasma lipids. Hearts from TTA-treated mice also showed a marked reduction in cardiac efficiency $(97 \%$ increase of unloaded $\mathrm{MVO}_{2}$, as deduced from the $\mathrm{y}$ intercept of the extrapolated $\mathrm{MVO}_{2}$ :PVA relationship). The gene expression analysis showed upregulation of PPAR $\alpha$ target genes, including mcptl, ctel, pdk4 and ucp3. The stimulatory effect of TTA on cardiac FA oxidation was not found in TTA-treated $\operatorname{PPAR} \alpha \mathrm{KO}$ mice. In conclusion, TTA is a potent transcriptional activator of 
$\operatorname{PPAR} \alpha$ in the heart, that markedly increases myocardial FA oxidation.

The Intrinsic Circadian Clock Within the Cardiomyocyte Directly Regulates Myocardial Gene Expression, Metabolism, and Contractile Function

Molly S. Bray, Chad A. Shaw, Michael W.S. Moore, Rodrigo A. P. Garcia, Melissa M. Zanquetta, Ju-Yun Tsai, Jason R. B. Dyck, Chi-Wing Chow and Martin E. Young. USDA/ARS Children's Nutrition Research Center, Baylor College of Medicine, Houston, Texas, 77030, USA.

Background - Virtually every mammalian cell, including cardiomyocytes, possesses an intrinsic circadian clock. The role of this transcriptionally-based molecular mechanism in cardiovascular biology remains unknown.

Hypothesis - We hypothesized that circadian clock within the cardiomyocyte plays a role in regulating myocardial metabolism and contractile function.

Methods and Results - To study the role of circadian clock within the heart, we generated a cardiomyocytespecific circadian clock mutant (CCM) mouse. Through microarray analysis, we identified 1155 and 362 clockregulated genes in atria and ventricles, respectively. These genes cluster into 5 major categories: regulation of transcription, transport, signal transduction, protein turnover, and metabolism. In addition, we observed circadian rhythms in cardiac power and efficiency in wild-type hearts ex vivo, which were abolished in CCM hearts. Moreover, myocardial oxygen consumption and fatty acid oxidation rates were increased, and heart rate was reduced in CCM hearts. Furthermore, CCM hearts demonstrated a loss of circadian rhythms in metabolic and functional responses to a workload challenge normally observed in wild-type hearts ex vivo.

Conclusions - These studies identify roles for the circadian clock within the cardiomyocyte, exposing direct influences on myocardial gene expression, metabolism, contractile function, and anticipation of workload changes.

\section{The Disastrous Effects of 8 Weeks of Overindulgence}

\section{B. Huisamen, D. Dietrich, S. Genade, A. Lochner Dept. Biomedical Sciences, Faculty of Health Sciences, University of Stellenbosch, Tygerberg,7505 (bh3@sun.ac.za)}

Background: Cardiovascular mortality is increased among persons with diabetes, and the incidence of heart failure, following a myocardial infarction is much greater among diabetic than non-diabetic patients. According to MRC predictions, the incidence of obesity is currently nearly $60 \%$ in some population groups in South Africa, foreshadowing an elevation in type 2 diabetes prevalence.

Aim and methods: We studied the effects of altered diet on myocardial function, using a rat model of hyperphagia $(\mathrm{DIO}=$ diet supplemented with sucrose and condensed milk for 8 weeks) compared to age-matched control animals. Afterwards, isolated perfused hearts were subjected to ischaemia/ reperfusion and infarct size determination by TTC staining. Fasting blood glucose, insulin and homocysteine levels and body weight were recorded.

Results: Body weights of DIO animals were significantly increased after 8 weeks on diet $(396.1 \pm 7.9$ vs $358.2 \pm 5.1$ gm, $p=0.0006, n=12$ ) and their fasting blood glucose levels significantly elevated $(4.65 \pm 0.2$ vs $3.78 \pm 0.1 p=$ $0.003, n=8)$. Baseline mechanical function was identical in both groups. However postischaemic functional recovery was significantly lower in DIO animals than in controls (41.9\% recovery vs $57.9 \% ; p<0.05, n=7-11)$. Surprisingly, hearts from the DIO animals presented with significantly smaller infarct size $(17.55 \pm 1.8 \%, p=0.005, n=8)$ than controls $(35.44 \pm 6.5 \%$ of the area at risk, $n=7)$ after regional ischaemia.

Conclusion: 8 weeks of DIO resulted in weight gain and elevated blood glucose levels. In conjunction with this, hearts showed curtailed functional recovery after global ischaemia but smaller infarct size after regional ischaemia indicating defects in contractility but probably not in survival pathways.

\section{'Western' Diet, but not High Fat Diet, Causes Maladaptation of Cardiac Fatty Acid Metabolism and Cardiac Dysfunction in the Wistar Rat}

Christopher R. Wilson ${ }^{1}$, Mai K. $\operatorname{Tran}^{1}$, Katrina L. Salazar $^{1}$, Femi Philip ${ }^{1}$, Joshua G. Leichman ${ }^{1}$, Patrick H. Guthrie $^{1}$, Terri M. King ${ }^{2}$, Martin E. Young ${ }^{3}$, Heinrich Taegtmeyer ${ }^{1}$

${ }^{1}$ Department of Internal Medicine - Division of Cardiology; University of Texas Medical School at Houston, University of Texas Health Science Center at Houston ${ }^{2}$ Department of Pediatrics; University of Texas Medical School at Houston, University of Texas Health Science Center at Houston ${ }^{3}$ Department of Pediatrics; USDA/ARS Children's Nutrition Research Center, Baylor College of Medicine

Obesity and diabetes are associated with increased fatty acid availability in excess of fatty acid oxidation capacity. This mismatch is implicated in the pathogenesis of cardiac contractile dysfunction. We tested the hypothesis that a "western" or a high fat diet will lead to maladaptation of cardiac fatty acid oxidation and subsequent contractile 
dysfunction. Male Wistar rats ( $n=12-17 /$ group) were fed a control, western, or high fat $(10 \%, 45 \%$, or $60 \%$ calories from fat, respectively) diet for acute (1 day and 1 week), short (1-2 months), intermediate (4-6 months), or long (812 months) term. In one set of rats, cardiac power, glucose oxidation and oleate oxidation were assessed in isolated working hearts. In parallel experiments, hearts were isolated for quantitative RT-PCR mRNA transcript analysis. Cardiac power decreased with the western diet $(-24 \%, p<$ $0.05)$. There was no significant decrease in cardiac power with the high fat diet. Oleate oxidation was increased with western diet in the acute $(+17 \%)$, short $(+36 \%)$ and intermediate term $(+31 \%)(p<0.01)$, but not in the long term. In contrast, oleate oxidation was increased with the high fat diet at all time points investigated $(+38 \%,+44 \%$, $+62 \%$, and $+15 \%$ for acute, short, intermediate, and long term, respectively; $p<0.05$ ). There was a sustained decrease in glucose oxidation with western diet by $38 \%(p<0.05)$ which was coincident with a sustained induction of the mRNA encoding for pyruvate dehydrogenase kinase 4 (PDK4) by $68 \%(p<0.01)$. Glucose oxidation was similarly decreased with high fat diet by $42 \%(p<0.05)$, which was coincident with a sustained induction of the mRNA encoding for PDK4 by $266 \%(p<0.01)$. mRNA transcripts for genes involved in fatty acid-mediated futile cycling, such as cytosolic thioesterase 1 (CTE-1), were upregulated to a greater extent by the high fat diet (CTE-1 average $+551 \%$, $p<0.001$ ), as compared to the western diet (CTE-1 average $+296 \%, p<0.001)$. In conclusion, a greater activation of oleate oxidation and PPAR $\alpha$-regulated gene expression in the hearts of rats fed a high fat diet, as compared to a western diet, is coincident with maintenance of cardiac function. This implicates inadequate activation of PPAR $\alpha$ with the western diet in the development of cardiac dysfunction.

\section{Metabolic Fluxes Quantitated from Pre-steady-state Single-time-point NMR Spectra of Mammalian Tissue Samples}

Johannes HGM van Beek, Johan Groeneveld, David JC Alders and Thomas Binsl

Centre for Integrative BioInformatics and Centre for Medical Systems Biology, VU University Amsterdam, the Netherlands

In this study metabolic fluxes in porcine heart in vivo are quantitated with a computational model for stable isotope distribution in the metabolic network. The experimental protocol entails exactly $5.5 \mathrm{~min}$ infusion in a coronary artery of carbon-13 enriched acetate which is metabolized in the TCA cycle. At the end of infusion, tissue samples from the heart are quickly frozen, extracted and carbon-13 NMR spectra determined. Flux parameters are estimated from the NMR multiplet intensities. Flux parameter optimization with the metabolic model for the TCA cycle and communicating amino acid pools yields 4 metabolic fluxes. The feasibility of flux quantitation and the optimal infusion time were demonstrated by computer simulations. Flux values were tested against 'gold standard' measurements of $\mathrm{O}_{2}$ consumption $\left(\mathrm{VO}_{2}\right)$. TCA cycle flux was quantified in absolute terms (mean \pm SD: $7.7 \pm 3.0 \mu \mathrm{mol} / \mathrm{g}$ dry weight $/ \mathrm{min}, 60$ samples from 7 normal pig hearts in situ) and agreed with the flux calculated by dividing $\mathrm{VO}_{2}$ by the stoichiometric ratio. NMR and $\mathrm{O}_{2}$ measurements on hearts in situ $(n=42)$ showed excellent correspondence $(r=0.88)$ over a broad range of conditions (baseline, dobutamine stress, ischemia, coronary vasodilation, lipopolysaccharide). The relation between local TCA cycle flux and blood flow was determined under all conditions. The linear relation between blood flow and TCA cycle flux explains $43 \%$ of blood flow variance in normal heart. At $870 \mu \mathrm{M}$ coronary venous acetate concentration, acetate delivers $58 \%$ of acetyl CoA taken up in the TCA cycle. This method makes it possible to quantitate fluxes in myocardial metabolic networks in vivo.

\section{Computational Model Analysis Of Dynamic Adaptation of Myocardial Oxidative Phosphorylation}

\section{Johannes HGM van Beek \\ Centre for Integrative BioInformatics and Centre for Medical Systems Biology, VU University Amsterdam, the Netherlands}

Cardiac workload fluctuates perpetuously and myocardial ATP synthesis adapts. A computational 'skeleton model' is analyzed for the first phase of dynamic adaptation of myocardial oxidative phosphorylation (OxPhos). This model centers on adenine nucleotides, creatine and phosphate groups. Well-known parameter values are included for the enzyme kinetics from cytoplasmic and mitochondrially located creatine kinase isoforms studied in isolation. The hotly debated mitochondrial outer membrane (MOM) permeability was estimated, using the model, from the dynamic response time of OxPhos $\left(\mathrm{t}_{\text {mito }}\right.$; generalized time constant) to jumps in cardiac pacing rate. The experimentally determined $t_{\text {mito }}$ is $3.7 \mathrm{~s}$ at the level of the mitochondria in rabbit heart with inhibited glycolysis and is reproduced with MOM permeability optimized to $21 \mu \mathrm{m}$ $\mathrm{s}^{-1}$ in the model. This value determined in vivo is about four times higher and four times lower, respectively, than the conflicting permeability values resulting from two different in vitro approaches, both incompatible with the measured $\mathrm{t}_{\text {mito }}$. The latter permeability values were compatible with ADP concentrations required for $50 \%$ stimulation of the 
mitochondria $\left(\mathrm{K}_{\mathrm{ADP}}\right)$ of about 20 and $250 \mu \mathrm{M} . \mathrm{K}_{\mathrm{ADP}}$ is therefore predicted to be about $70 \mu \mathrm{M}$ in vivo. The model predicts correctly that a lower $t_{\text {mito }}$ is measured if $\mathrm{CK}$ activity is inhibited by $98 \%$. The model predicts further that myocardial ATP synthesis shows substantial pulsatility, that OxPhos reacts slower if $\mathrm{CK}$ in the cytoplasm is overexpressed and faster if mitochondrial $\mathrm{CK}$ is overexpressed. About one third of the transport of 'high energy' phosphoryl groups in rabbit cardiomyocytes is via phosphocreatine.

\section{TIME-Resolved Analysis of NAD(P)H in Living Cardiac Cells: The Effect of 4-Hydroxynonenal (HNE)}

Aneba $\mathrm{S}^{1}$, Chorvat $\mathrm{D} \mathrm{Jr}^{2}$, Mateasik $\mathrm{A}^{2}$, Comte $\mathbf{B}^{1,3}$, Chorvatova $\mathrm{A}^{1,4}$

${ }^{1}$ Research Centre of CHU Sainte-Justine, University of Montreal, Montreal, Canada; ${ }^{2}$ International Laser Centre, Bratislava, Slovakia; Departments of ${ }^{3}$ Nutrition and ${ }^{4}$ Pediatrics, University of Montreal, Montreal, Canada.

$\mathrm{NAD}(\mathrm{P}) \mathrm{H}$ plays a crucial role in the management of oxidative metabolism as the principal electron donor for enzymatic reactions and a major source of autofluorescence induced following excitation by UV light. Screening fluorescence dynamics of intrinsic cellular autofluorescence is a powerful tool to investigate cardiomyocyte metabolic and oxidative properties. Our aim is to develop a new approach of highcontent analysis of $\mathrm{NAD}(\mathrm{P}) \mathrm{H}$ autofluorescence assessing oxidative metabolic changes in living cardiomyocytes and testing the cellular $\mathrm{NAD}(\mathrm{P}) \mathrm{H}$ responses to $\mathrm{HNE}$, an important by-product of lipid peroxidation. Spectrally-resolved timecorrelated single photon counting (TCSPC) was used to measure $\mathrm{NAD}(\mathrm{P}) \mathrm{H}$ fluorescence spectra and fluorescence lifetimes following excitation by a pulsed picosecond $375 \mathrm{~nm}$ laser diode. Freshly isolated living cardiomyocytes were treated with HNE in the presence or absence of a glutathione reductase blocker, or of complex I and IV respiratory chain inhibitors. In agreement with the capacity of HNE to decrease NADPH production by inhibiting NADP-isocitrate dehydrogenase, treated cardiomyocytes presented a loss of $\mathrm{NAD}(\mathrm{P}) \mathrm{H}$ autofluorescence, accompanied by a slight red spectral broadening. Our data uncovered implication of the enzymes of the respiratory chain in the HNE action; results in contrast with effects of $\mathrm{H}_{2} \mathrm{O}_{2}$, a common oxidative agent, point to implication of different metabolic pathways.

Our findings indicate that spectrally-resolved TCSPC is a powerful tool for quantitative high content assays of NAD(P) $\mathrm{H}$ molecules in living cardiomyocytes. It can be particularly useful for understanding the role of mitochondrial metabolism and oxidative stress in the development of cardiac diseases. Supported by the Canadian Institutes for Health Research.
AMPK-mediated Increase in Myocardial Long-Chain Fatty Acid Uptake Critically Depends on Sarcolemmal CD36

Daphna D.J. Habets, ${ }^{1}$ Will A. Coumans, ${ }^{1}$ Peter J. Voshol, ${ }^{2}$ Marion A.M. den Boer, ${ }^{2}$ Maria Febbraio, ${ }^{3}$ Arend Bonen, ${ }^{4}$ Jan F.C. Glatz, ${ }^{1}$ Joost J.F.P. Luiken ${ }^{1,5}$

${ }^{1}$ Department of Molecular Genetics, Cardiovascular Research Institute Maastricht (CARIM), Maastricht University, Maastricht, the Netherlands ${ }^{2}$ Department of Endocrinology and Diabetes, Leiden University Medical Center, Leiden, the Netherlands ${ }^{3}$ Departments of Cell Biology and Molecular Cardiology, the Center for Cardiovascular Diagnostics and Prevention, Lerner Research Institute, Cleveland Clinic Foundation, Cleveland, $\mathrm{OH}$, USA ${ }^{4}$ Department of Human Health and Nutritional Sciences, University of Guelph, Guelph, Ontario N1G 2W1, Canada ${ }^{5}$ Department of Biochemical Physiology and Institute of Biomembranes, Utrecht University, Utrecht, the Netherlands

CD36, also named fatty acid translocase, has been identified as a putative membrane transporter for longchain fatty acids (LCFA). In the heart, contraction regulates cellular LCFA uptake through translocation of CD36 and possibly of other LCFA transporters from intracellular storage compartments to the sarcolemma. This regulation is mediated by contraction-induced 5' AMP-activated protein kinase (AMPK) signaling. In this study isolated cardiac myocytes from $\mathrm{CD} 36^{+/+}$and $\mathrm{CD} 36^{-/-}$mice were used to investigate to what extent basal and AMPKmediated LCFA uptake are CD36-dependent.

Basal LCFA uptake was not altered in $\mathrm{CD} 36^{-/-}$mice, likely because of a (1.8-fold) compensatory upregulation of fatty acid transport protein-1. The CD36 inhibitor sulfo$N$-succinimidylpalmitate reduced basal LCFA uptake into cardiac myocytes from $\mathrm{CD} 36^{+/+}$mice by $38 \%$, while not affecting cardiac myocytes from $\mathrm{CD} 36^{-/-}$mice. Oligomycin and dipyridamole, two contraction-like stimuli, increased LCFA uptake into cardiac myocytes from $\mathrm{CD} 36^{+/+}$mice by 2.5- and 1.6-fold, respectively. In contrast, in cardiac myocytes from $\mathrm{CD} 36^{-/-}$mice, oligomycin and dipyridamole increased LCFA uptake only by 1.3- and 1.1-fold, respectively, despite the fact that AMPK signaling in these cardiac myocytes was fully intact. Glucose uptake was not different between cardiac myocytes from $\mathrm{CD} 36^{+/+}$and $\mathrm{CD} 36^{-/-}$mice under basal conditions nor in the presence of oligomycin or dipyridamole.

We conclude that CD36 is almost entirely responsible for the AMPK-mediated increase of LCFA uptake, indicating a pivotal role for $\mathrm{CD} 36$ in mediating changes in cardiac LCFA fluxes. 


\section{Energy Metabolism in Cultured Adult Rat Cardiac Myocytes}

Wino J. Wijnen, Daphna D.J. Habets, Jan F.C. Glatz, Joost J.F.P. Luiken

Department of Molecular Genetics; CARIM, Maastricht University, the Netherlands

Cardiac myocytes need a constant supply of energy. The main cardiac energy substrates are fatty acids (FA) and glucose. Their uptake occurs in a regulated process, involving several transport proteins (GLUT1 and GLUT4 for glucose, and CD36 and several other FA-binding proteins for FA) and signal transduction cascades (insulin signaling, AMPK signaling, etc). For both GLUT4 and CD36, activation of signal transduction cascades results in translocation from an intracellular pool to the sarcolemma, where the transporters increase the rate of substrate uptake.

Type 2 diabetes mellitus (DM2) leads to altered energy substrate utilization. DM2 is characterized by insulin resistance, increased FA utilization and a permanent relocation of CD36 to the sarcolemma.

We aim at studying the early onset of DM2 in a suitable model system. Cultures of adult primary rat cardiac myocytes have many characteristics making them a good model system. Upon isolation these cells have the phenotype of the cells of interest, while in culture the environment can be controlled. Cultured cells can be electrically stimulated to controlled contractions and can be infected with modified viruses to manipulate the expression of selected proteins. On the other hand, because the cells do not divide, studies are limited to several days due to cell losses and adaptation resulting in changes at the morphological and metabolic level.

Preliminary data on the short-term culturing of adult primary rat cardiac myocytes will be presented.

Supported by the Netherlands Organization for Health Research and Development (NWO-ZonMw grant 40 00812-98-03075)

\section{RAB11a is Short-time Activated by Insulin and Regulates the Internalization of GLUT4 and FAT/CD36 Containing Vesicles}

\author{
R.W. Schwenk ${ }^{1}$, J.J.F.P Luiken ${ }^{2}$, J. Eckel ${ }^{1}$ \\ ${ }^{1}$ Institute of Clinical Biochemistry and Pathobiochemistry, \\ German Diabetes Center, Düsseldorf, Germany; ${ }^{2}$ Department \\ of Molecular Genetics, Cardiovascular Research Institute \\ Maastricht (CARIM), Maastricht University, Maastricht, \\ The Netherlands
}

As a member of the Rab small GTPase family, Rab11a has been shown to be involved in different vesicle trafficking processes. In earlier work we identified Rab1la to be present in GLUT4 and in FAT/CD36 containing vesicles, with an increase in GLUT4 containing vesicles after insulin stimulation. However, it remained elusive if Rab11a is directly activated by the insulin signalling cascade and at which step a potential activation occurs. To examine the GTP-loading of Rab11a, we introduced a biotinylated GTP-analog into H9c2hIR cells, transiently overexpressing HA-tagged Rab11a, and measured its binding to the GTPase after insulin stimulation. We observed that Rab11a is transiently GTP-loaded after insulin stimulation. The activation of Rab1la is PI3K dependent and downstream of Akt, as shown by in vitro knockdown of this kinase. To further specify the functional role of Rab11a in vesicle movement of GLUT4 and FAT/ CD36 we knocked down gene expression of Rab11a and two of its effectors, Rip11 and FIP2, by small interfering RNAs (siRNAs) and measured translocation of GLUT4myc and FAT/CD36. We observed that by knocking down Rab11a GLUT4myc- and FAT/CD36-translocation is increased as well in the basal as in the insulin (for GLUT4) and sorbitol (for FAT/CD36) stimulated state. In the case of GLUT4myc the in vitro knockdown of FIP2 increased the translocation even stronger than the Rab11a knockdown, whereas Rip11 knockdown had no effect. The FAT/CD36-translocation is slightly increased after Rip11 knockdown and to a larger amount after FIP2 knockdown, but not as strong as after Rab11a gene repression. In both vesicle populations the effect of the stimulus on translocation is not altered after gene knockdown of Rab11a.

These data therefore show that (i) Rab11a is directly activated by insulin and represents an element of the PI3K signalling cascade, (ii) Rab1la regulates the endocytosis of both, GLUT4 and FAT/CD36 containing vesicles, (iii) the regulation of GLUT4 endocytosis by Rab11a is carried out in complex with Rab11-FIP2 and differently to FAT/CD36.

Supported by the Ministerium für Wissenschaft und Forschung des Landes Nordrhein-Westfalen, the Bundesministerium für Gesundheit and European Union COST Action B17.

\section{Electromagnetic Sensors of Metabolic Activity}

John H. Miller, Jr., ${ }^{1}$ Jie Fang, ${ }^{1}$ Shih-Ying Hsu, ${ }^{1}$ William R. Widger, ${ }^{2}$ and Dale J. Hamilton ${ }^{3 *}$

${ }^{I}$ Dept. of Physics and Texas Ctr. for Superconductivity, Univ. of Houston, Houston, TX 77204-5005 ${ }^{2}$ Dept. of Biology and Biochemistry, University of Houston, Houston, Texas 77204-5001 ${ }^{3}$ Dept. of Medicine, The Methodist Hospital, 6550 Fannin St., Suite 1001, Houston, Texas 77030 
Background: The human heart recycles $\sim 35 \mathrm{~kg}$ of ATP in a single day. Thus, cardiac metabolism is emerging as a target for the treatment of heart failure, and mitochondrial dysfunction is becoming implicated in heart disease.

Hypothesis/objectives: Oscillatory electric fields induce membrane proteins to change conformation leading to nonlinear responses of charged residues and ions, which results in the generation of harmonics that can be picked up by sensing electrodes. Our objectives are to develop and study electromagnetic methods [1] that probe metabolic activity of enzyme complexes in mitochondria, the cellular plasma membrane, and other internal membranes.

Methods: We measure $2^{\text {nd }}$ and higher degree harmonics generated by whole cells, mitochondria, and chloroplasts, in response to $\mathrm{AC}$ electric fields at frequencies ranging up to a few ten's of kilohertz.

Results: The measured harmonics depend strongly on frequency, field amplitude, and metabolic activity, as shown in Fig. 1. In the case of mitochondrial suspensions, electron transport chain (ETC) activators and inhibitors affect observed features in $2^{\text {nd }}$ harmonic spectra.

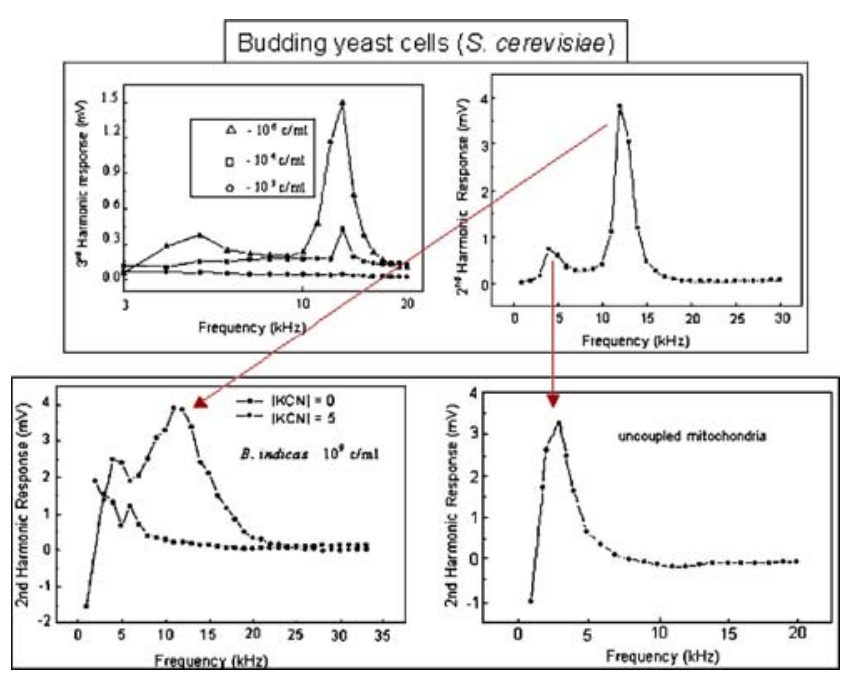

Harmonic generation spectra of suspensions of budding yeast ( $S$. cerevisiae, top), a relative of the mitochondrial ancestor (B. indicas, lower left), and uncoupled mammalian mitochondria, in which ETC complexes I, III, and IV have been activated by adding glutamate malate (lower right). The spectral features generated by $S$. cerevisiae are observed to increase with cell concentration (upper left). We find that potassium cyanide, which binds to complex IV and completely shuts down ATP production, suppresses the observed spectral features, as seen in the $B$. indicas data. The higher frequency feature $(\sim 12 \mathrm{kHz})$ observed in whole cells is not seen in uncoupled mitochondria, where ATP synthase is inactive. The data was obtained with the 4-electrode setup, in which a sinusoidal voltage is applied across the outer two electrodes and the induced harmonics are measured across two inner electrodes with a spectrum analyzer.

Conclusions: Harmonic generation spectroscopy reflects mitochondrial ETC function in live cells, and provides a technique to assess mitochondrial function in human tissues. A possible application includes assessment of myocardial mitochondria in patients with heart failure and diabetes. We are also exploring methods to electromagnetically probe enzyme activity at the nanoscale.

[1] Miller, JH, Jr. et al. Electromagnetic probes of molecular motors in the electron transport chains of mitochondria and chloroplasts. $J$ de Physique IV France, 2005;131:363-366.

\section{TGF- $\beta$ and 12-Lipoxygenase Regulate Glut4 Expression in Cardiac Cells}

${ }^{1}$ Swifka J., ${ }^{2}$ Sasson S. and ${ }^{1}$ Eckel J.

${ }^{1}$ Institute of Clinical Biochemistry and Pathobiochemistry, German Diabetes Center, Düsseldorf, Germany; ${ }^{2}$ Hebrew University, School of Medicine, Jerusalem, Israel

We have recently shown that eicosanoids participate in the regulation of cardiac glucose transport and GLUT4 translocation. In the present study we investigated the role of 12-lipoxygenase (12-LO) in the regulation of glucose transporter 4 (GLUT4) expression and its translocation to the plasma membrane in a cardiac cell system. We established a robust gene knock down of 12-LO by siRNA in H9c2-hIR cells. After siRNA treatment for $48 \mathrm{~h}$ we could downregulate 12-LO protein expression to $30 \pm 5 \%(p<0.001)$ and mRNA expression to $10 \pm 4 \%(p<0.01)$. In addition, treatment with baicalein, a 12-LO specific inhibitor, decreased 12-LO protein levels to $40 \pm 5 \%(p<0.05)$.

Generation of 12(S)-hydroxyeicosatetraenoic acid (HETE), a 12-LO specific product and inflammatory mediator, was affected by 12-LO abundance as measured with a HETE ELISA $(n=5)$. After siRNA mediated gene knockdown of 12-LO (si 12-LO), HETE expression was reduced to $50 \pm$ $10 \%(p<0.001)$ normalized to incubation with non coding siRNA (nc siRNA). 12-LO inhibition decreased HETE to $55 \pm 15 \%(p<0.05)$ in comparison to untreated cells (uc). Compared to this data HETE generation was significantly increased after 12-LO activation by TGF- $\beta(144 \pm 10 \%$; $p<$ $0.05)$ or high glucose $(125 \pm 15 \% ; p<0.05)$ over night. However, TGF- $\beta$ could not increase HETE generation after gene knockdown of 12 -LO $(75 \pm 5 \% ; p<0.05)$ compared to nc siRNA.

In a parallel approach we overexpressed a myc epitopetagged GLUT4. GLUT4 protein expression was significantly 
elevated up to $420 \pm 70 \%(p<0.01)$ in si12-LO transfected cells in comparison to transfection with nc siRNA. This effect was 12-LO specific, because transfection with siAkt (90\% knockdown), does not change GLUT4 protein expression compared to nc siRNA. 12-LO downregulation also affected GLUT4 mRNA expression in accordance with our results at the protein level. Consistent to these findings, an incubation with TGF- $\beta$ over night down regulated GLUT4 protein expression in uc and nc siRNA transfected cells. However, when silencing 12-LO, the effect of TGF- $\beta$ on GLUT4 protein expression was no more detectable $(p<$ 0.05). In H9c2-hIR cells overexpressing GLUT4, 2-DOG uptake and GLUT4 translocation can be stimulated by insulin up to $150 \pm 10 \%(p<0.001)$ and $170 \pm 15 \%(p<$ 0.001 ), respectively. 12 - LO knock down totally abolished insulin stimulated 2-DOG uptake ( $>0.05)$, despite higher GLUT4 expression. Accordingly insulin - stimulated GLUT4 translocation to the plasma membrane was completely inhibited after 12 - LO downregulation ( $p>0.05$ ).

In conclusion eicosanoids appear to be regulators of GLUT4 expression and it might be hypothesized that increased eicosanoid levels during inflammation may cause GLUT4 down regulation. We demonstrated that 12-LO repression causes a defect in insulin - induced translocation of GLUT4 to the plasma membrane and 2DOG uptake. This can possibly be attributed to a loss in HETE production and thereby a destabilisation of the cytoskeleton. (This work was supported by German Israel Foundation)

\section{Insulin Induces Reversible Translocation of CD36 to the Plasma Membrane in Cho Cells: a Morphological Characterization}

Masja M. van Oort ${ }^{1}$, Jan M. van Doorn ${ }^{1}$, Arend Bonen ${ }^{2}$, Jan F.C. Glatz ${ }^{3}$, Dick J. van der Horst ${ }^{1}$, Kees W. Rodenburg ${ }^{1}$, and Joost J.F.P. Luiken ${ }^{1,3}$

${ }^{1}$ Division of Endocrinology and Metabolism, Department of Biology and Institute of Biomembranes, Utrecht University, NL-3584 CH Utrecht, the Netherlands ${ }^{2}$ Department of Human Health and Nutritional Sciences, University of Guelph, Guelph, Ontario N1G 2W1, Canada ${ }^{3}$ Department of Molecular Genetics, Cardiovascular Research Institute Maastricht (CARIM), Maastricht University, NL-6200 MD Maastricht, the Netherlands

In cardiac and skeletal muscle, long-chain fatty acid (LCFA) uptake is regulated by insulin via the putative LCFA transporter CD36. Biochemical studies propose an insulin-induced translocation of CD36 from intracellular, endosomal pools to the plasma membrane (PM), similar to translocation of glucose transporter 4 (GLUT4). To morphologically characterize insulin-induced CD36 translocation, we developed Chinese hamster ovary $(\mathrm{CHO})$ cells stably expressing rat CD36 (CHO-CD36). Using immunofluorescence microscopy, CD36 was found both intracellularly - in (at least partially) different compartments than GLUT4 - and at the PM, and translocated to the PM upon insulin incubation, as did myc-tagged GLUT4 (GLUT4myc). The insulin-induced increase in cell surface CD36 content was confirmed and quantified by surface-protein biotinylation (1.7-fold increase). Moreover, insulin-induced CD36 translocation was wortmannin-sensitive (i.e. phosphatidylinositol-3 kinase-dependent), and reversible (30-60 minutes after insulin wash-out). Hence, insulin-induced CD36 translocation in $\mathrm{CHO}$ cells closely resembles the proposed physiological process in skeletal muscle and heart. Whereas the insulin-induced increase in PM GLUT4myc correlates with an increased $\left[{ }^{3} \mathrm{H}\right]$ deoxyglucose uptake into $\mathrm{CHO}$ cells, the increase in PM CD36 content was not paralleled by a change in $\left[{ }^{14} \mathrm{C}\right]$ palmitate uptake, suggesting that additional proteins or a protein activation step are needed. Taken together, this study presents the first morphological evidence for CD36 translocation, and advocates $\mathrm{CHO}-\mathrm{CD} 36$ cells as an adequate model system to further characterize this process.

Skeletal Muscle Fatty Acid Transporter Protein Expression in Type 2 Diabetes Patients Compared with Overweight, Sedentary Men and Age-matched, Endurance-trained Cyclists

M.M.A.L. Pelsers ${ }^{1}$, K. Tsintzas ${ }^{3}$, H. Boon ${ }^{2}$, K. Jewell ${ }^{3}$, L. Norton ${ }^{3}$, J.J.F.P. Luiken ${ }^{4}$, J.F.C. Glatz ${ }^{4}$, and L.J.C. van Loon ${ }^{1,2}$

Departments of Movement Sciences ${ }^{1}$, Human Biology ${ }^{2}$, and Molecular Genetics ${ }^{4}$, Maastricht University, Maastricht, the Netherlands. Institute of Clinical Research ${ }^{3}$, University of Nottingham Medical School, Nottingham, United Kingdom.

Aim Membrane fatty acid transporters can modulate the balance between fatty acid uptake and subsequent storage and/or oxidation in muscle tissue. As such, skeletal muscle fatty acid transporter protein expression could play an important role in the etiology of insulin resistance and/or type 2 diabetes.

Methods In the present study fatty acid translocase (FAT/ CD36), plasma membrane-bound fatty acid-binding protein (FABPpm) and fatty acid transport protein 1 (FATP1) mRNA and protein expression were assessed in muscle 
tissue obtained from 10 sedentary, overweight type 2 diabetes patients $(60 \pm 2 \mathrm{yrs}), 10$ sedentary, weight-matched normoglycemic controls ( $60 \pm 2 \mathrm{yrs})$ and 10 age-matched, endurance trained cyclists ( $57 \pm 1 \mathrm{yrs})$.

Results Both FAT/CD36 and FATP1 mRNA and protein expression did not differ between groups. In contrast, FABPpm mRNA and protein expression were $\sim 30-40 \%$ higher in the trained men compared to the diabetes patients $(P<0.01)$ and sedentary controls $(P<0.05)$.

Conclusions Skeletal muscle FAT/CD36, FABPpm and FATP1 mRNA and protein expression are not up- or down regulated in a sedentary and/or insulin resistant state. In contrast, FABPpm expression is upregulated in the endurance trained state and likely instrumental to allow greater fatty acid oxidation rates.

\section{Characterizing the PPAR $\alpha$ Dependent Transcriptome in Murine Heart}

Anastasia Georgiadi, Michael Müller, Sander Kersten
Nutrition, Metabolism and Genomics Group, Division of
Human Nutrition, Wageningen University, the Netherlands

The peroxisome proliferators-activated receptor alpha is a ligand activated transcription factor activated by a variety of fatty acids and lipophilic compounds. Studies in liver have revealed that PPAR $\alpha$ governs a variety of metabolic processes, including fatty acid catabolism and inflammation. Although PPAR $\alpha$ is expressed at high levels in the heart, there is little knowledge on the type of pathways that are under control of PPAR $\alpha$ in heart.

The aim of our study is to provide a global overview of genes regulated by PPAR $\alpha$ in the heart. For that purpose we used a highly specific PPAR $\alpha$ synthetic ligand Wy14643. Wildtype and PPAR $\alpha^{-/-}$mice were fed a diet supplemented with $0.1 \%$ Wy 14643 , for 5 days. Total RNA from heart was hybridized on Affymetrix NuGO customized arrays. Normalization was performed by GC-RMA and differentially expressed transcripts were selected based on fold-change cutoff. Pathway analysis tools were utilized to identify metabolic processes regulated by Wy14643.

Five days feeding with Wy14643 increased the expression of 339 genes and decreased the expression of 302 genes, none of which were altered in PPAR $\alpha^{-/-}$mice. Pathways analysis revealed that various fatty acid metabolic pathways were under control of PPAR $\alpha$.

In conclusion, we show by microarray analysis that $\operatorname{PPAR} \alpha$ plays an important role in the regulation of fatty acid metabolism in heart. A more in depth analysis of pathways regulated by PPAR $\alpha$ and of the effect of dietary fatty acid on global cardiac gene expression is ongoing.

Supported by the European-Marie Curie project 'NucSys'.

\section{Upregulation of Lipoprotein Secretion: A Mechanism to Maintain Myocardial Lipid Homeostasis in Cardiomyocyte-restricted PPAR $\delta$ Knockout Mice?}

Qinglin Yang, Qianhong Qin, Jian Liu, Yuquan Li, Ran Yin, Eva Madsen, Lars B. Nielsen

Dept. of Biochemistry, Cardiovascular Research Institute, Morehouse School of Medicine, Atlanta GA, USA

Peroxisome proliferator-activated receptors are nuclear hormone receptors and ligand-activated transcription factor controlling lipid and carbohydrate metabolisms. All three subtypes of PPARs, PPAR $\alpha, \gamma$ and $\delta$ are expressed in the heart. They may serve as key determinants of myocardial fatty acid metabolism. We previously demonstrated that PPAR $\delta$ is required for normal expression of series essential enzymes that govern myocardial fatty acid oxidation (FAO). Cardiomyocyte-restricted PPAR $\delta$ knockout in mice $\left(\mathrm{CR}-\mathrm{PPAR} \delta^{-/}\right.$) shows depressed myocardial FAO and bioenergetics, leading to dilated cardiomyopathy with myocardial lipid accumulation at the end stage hearts. It remains unclear why myocardial triglyceride content is largely normal in young adult CR-PPAR $\delta^{-/-}$hearts, despite a depressed myocardial FAO rate. We further investigate lipid uptake and lipoprotein secretion pathways in the mouse hearts of the cardiomyocyte-restricted PPAR $\delta$ knockout mice. Using Quantitative real-time PCR (QPCR), we discovered that transcript levels of genes encoding major fatty acid uptake proteins such as CD36, fatty acid transport protein-1, fatty acid biding protein, were downregulated by about 33, 46 and 50\%, respectively, in CR-PPAR $\delta^{-/-}$hearts compared with controlled hearts. Interestingly, the protein expression levels of these fatty acid uptake proteins were not correspondingly decreased in the adult CR-PPAR $\delta^{-/-}$hearts. Since lipoprotein mediated triglyceride secretion from cardiomyocytes may play a role in maintaining myocardial lipid homeostasis, we further assessed transcript expression levels of genes encoding proteins that are crucial for lipoprotein secretion from cardiomyocytes. QPCR revealed that the expression levels of Microsomal triglyceride transfer protein (MTP), the protein required to add lipid to apolipoprotein $\mathrm{B}(\mathrm{ApoB})$, were attenuated about $36 \%$ in CR-PPAR $\delta^{-/-}$hearts compared with controls, whereas the expression levels of apoB transcript were increased over 10 fold in CR-PPAR $\delta^{-/}$hearts. The corresponding protein levels of apoB were substantially increased in CR-PPAR $\delta^{-/-}$hearts compared with controls. Despite the downregulation of MTP transcritp, MTP activities 
were substantially increased in CR-PPAR $\delta^{-/-}$hearts compared to controls. These results implicate that CR-PPAR $\delta^{-/-}$hearts maintain relative myocardial lipid homeostasis via upregulating apoB expression and MTP activities to increase lipoprotein secretion, thus helping the young CR-PPAR $\delta^{-/-}$ hearts to avoid early accumulation of neutral lipid.

PDK1, but not PKB, is Required for the Activation of the Mtor/P70s6k Pathway by Leucine in the Heart

C. Sanchez Canedo ${ }^{1}$, C. Beauloye ${ }^{1}$, B. Demeulder ${ }^{1}$, A. Ginion ${ }^{1}$, E. Zarrinpashneh ${ }^{1}$, D.R. Alessi ${ }^{3}$, L. Hue ${ }^{2}$, J.-L. Vanoverschelde ${ }^{1}$ and L. Bertrand ${ }^{1}$

${ }^{1}$ Division of Cardiology \& ${ }^{2}$ Hormone and Metabolic Research Unit, Université catholique de Louvain, Brussels, Belgium. ${ }^{3}$ MRC Protein Phosphorylation Unit, University of Dundee, Dundee, Scotland.

Background: Insulin regulates the mammalian target of rapamycine/p70 S6 ribosomal protein kinase (mTOR/ p70S6K) pathway through a canonical signalling pathway that includes the phosphoinositide-dependent protein kinase 1/ protein kinase $\mathrm{B}$ (PDK1/PKB) axis. Currently, the mechanism of amino acid input integrated with that of the insulin signalling pathway is unclear.

Aim: We examined signalling events that activate p70S6K in response to insulin and amino acids in the adult heart.

Methods: Hearts from adult wild-type (WT) and cardiospecific PDK1 knock-out (KO) mice were isolated and perfused by the Langhendorff method in normoxic conditions. Results: In WT hearts, insulin and leucine significantly increased p70S6K activity (from $1.31 \pm 0.35 \mathrm{mU} / \mathrm{mg}$ prot to $7.84 \pm 0.94$ and $4.52 \pm 1.03 \mathrm{mU} / \mathrm{mg}$ prot, respectively). By contrast, glutamine inhibited p70S6K activity both in the absence $(0.59 \pm 0.22 \mathrm{mU} / \mathrm{mg}$ prot, $p<0.05)$ and in presence of insulin ( $3.39 \pm 0.58 \mathrm{mU} / \mathrm{mg}$ prot, $p<0.05)$. Moreover, insulin and leucine but not glutamine induced mTOR phosphorylation in WT hearts. As expected, insulin activated PKB $(1.89 \pm 0.34$ vs. $0.74 \pm 0.08 \mathrm{mU} / \mathrm{mg}$ prot in control heart, $p<0.05)$. Nevertheless, neither leucine nor glutamine was able to activate PKB. In PDK1 KO mice, the insulin and leucine effect on p70S6K activity and mTOR phosphorylation was abolished. The insulin- and leucineinduced p70S6K activation was differentially affected by use of the PI3K inhibitors: wortmannin and LY294002, suggesting the implication of different class of PI3K.

Conclusion: In contrast to insulin, leucine activates p70S6K via a PI3K-like/PDK1/mTOR-dependent but PKB-independent pathway.

\section{Regulation of MLCK by AMPK and P38}

Sandrine Horman ${ }^{1}$, Nicole Morel $^{2}$, Didier Vertommen ${ }^{1}$, Christophe Beauloye ${ }^{3}$, Nicole El Najjar ${ }^{1}$, Benoit Viollet ${ }^{4}$, Michael Walsh ${ }^{5}$, Louis Hue ${ }^{1}$ and Mark Rider ${ }^{1}$.

${ }^{1}$ Hormone and Metabolic Research Unit, Christian de Duve Institute of Cellular Pathology, ${ }^{2}$ Laboratory of Pharmacology, ${ }^{3}$ Division of Cardiology, Université catholique de Louvain, Brussels, Belgium ${ }^{4}$ U-567 INSERM UMR 8104 CNRS, René Descartes University, Institut Cochin, Department of Genetics, Development and Molecular Pathology, Paris, France ${ }^{5}$ Smooth Muscle Research Group and Department of Biochemistry and Molecular Biology, University of Calgary, Faculty of Medicine, 3330 Hospital Drive N.W., Calgary, Alberta, Canada, T2N $4 N 1$

Background. Contraction of vascular smooth muscle can be initiated by a number of stimuli inducing a rise in intracellular calcium and the subsequent stimulation of myosin light chain kinase (MLCK) activity, which, in turn, phosphorylates myosin light chain (MLC). The MLCK is also regulated by phosphorylation leading to modification of $\mathrm{K}_{0.5}$ for $\mathrm{Ca}^{++}$or Vmax values. Hypothesis. This study showed that MLCK was a new substrate for p38 mitogen-activated protein kinase (p38) and for AMP-activated protein kinase (AMPK). We tested the hypothesis that both kinases would be key regulators of MLCK in vascular smooth muscle (VSM).

Methods and Results. In vitro phosphorylation of chicken gizzard purified MLCK by recombinant AMPK allowed us to identify one phosphorylation site located in the $\mathrm{C}$-terminal $\mathrm{Ca}$ $++/$ calmodulin domain of MLCK. This phosphorylation led to an increased $\mathrm{K}_{0.5}$ for $\mathrm{Ca}^{++}$. By contrast, MLCK phosphorylation by p38 resulted in an increased Vmax due another phosphorylation site. Interestingly, phosphorylation at both sites resulted in MLCK activation suggesting the inhibitory effect of the AMPK site is blunted by the p38 phosphorylation site. In primary cultures of VSM cells, AMPK and p38 were activated in response to vasoconstrictors within the same time. When the AMPK pathway was inhibited using specific inhibitors, p38 was over activated.

Conclusion. We proposed that AMPK negatively regulates p38 and MLCK in vascular smooth muscle. Results obtained with alpha-1 knockout mice support this conclusion and will be presented at the meeting.

\section{Regulation of Glucose Uptake in Cardiomyocytes by Amp-activated Protein Kinase}

Christopher T. Lee, John Edward R. Ussher, Gary D. Lopaschuk

Cardiovascular Research Group, Departments of Pediatrics and Pharmacology, University of Alberta, Canada 
Glucose uptake and glycolysis in the heart are increased during myocardial ischemia in order to provide an additional anaerobic source of ATP for the heart. This alteration in glucose metabolism constitutes an important contributing factor towards ischemic injury, and therefore it is important to understand how glucose uptake is controlled in the ischemic heart. There are primarily two glucose transporters which regulate glucose uptake into cardiac myocytes, GLUT1 and GLUT4. In the non-ischemic heart, insulin is only able to stimulate the translocation of GLUT4 to the sarcolemmal membrane, while both GLUT1 and GLUT4 translocation can occur following AMPK stimulation. Using a newly developed technique involving $\left[{ }^{3} \mathrm{H}\right] 2$ deoxyglucose, we measured glucose uptake in the $\mathrm{H} 9 \mathrm{c} 2$ rat cardiomyocyte cell line, and demonstrate that while insulin has no detectable effect on glucose uptake, AMPK stimulation by phenformin results in a 2.5 -fold increase in glucose uptake. Furthermore, Insulin treatment produced no discernible effect on either phospho-Akt or phosphoAMPK intracellular protein levels, while treatment with phenformin results in an increase in phospho-AMPK, and a decrease in phospho-Akt. Finally, visualization of a dsRedGLUT4 fusion construct in H9c2 cells by laser confocal microscopy showed that unlike insulin, AMPK stimulation with phenformin did not redistribute GLUT4 to the plasma membrane, suggesting that AMPK may be regulating glucose uptake via another glucose transporter. These studies suggest that AMPK is a major regulator of glucose uptake in cardiomyocytes.

\section{UCP3 and Mitochondrial ROS Production in Cardiac and Skeletal Muscle}

Miranda Nabben ${ }^{\mathrm{a}}$, Joris Hoeks ${ }^{\mathrm{a}}$, Jan F.C. Glatz ${ }^{\mathrm{b}}$, Jacco J. Briede $^{\mathrm{c}}$, Matthijs K.C. Hesselink ${ }^{\mathrm{d}}$, Patrick Schrauwen ${ }^{\mathrm{a}}$ Departments of ${ }^{a}$ Human Biology, ${ }^{c}$ Health Risk Analysis and Toxicology, and ${ }^{d}$ Movement Sciences, Nutrition and Toxicology Research Institute Maastricht (NUTRIM), Maastricht University; ${ }^{b}$ Dept of Molecular Genetics, Cardiovascular Research Institute Maastricht (CARIM), Maastricht University, the Netherlands

Background: UCP3, which is present in the inner membrane of cardiac and skeletal muscle mitochondria, is increased when fatty acid (FA) supply to the mitochondria exceeds FA oxidation and is decreased in situations where fat oxidation exceeds FA supply. Remarkably, UCP3 is downregulated in type II diabetic patients, and pre-diabetic subjects. However, the exact function of the protein is still unknown.

Hypothesis/objectives: Previous results suggested involvement of UCP3 in the protection of mitochondria against excessive fatty acids, thereby preventing lipid-induced mitochondrial damage. To test this hypothesis, the effect of UCP3 overexpression on Reactive Oxygen Species (ROS) production was investigated.

Methods: Both cardiac and skeletal muscle mitochondria were isolated from 23-week-old male C57B16 mice overexpressing UCP3 (UCP3Tg) $(n=5)$, and their wildtype (Wt)controls $(n=4)$. ROS production was measured using Electron Spin Resonance spectroscopy after addition of spin trap (DMPO). Pyruvate was used as glycolytic substrate for cardiac measurements, whereas glutamate, malate (classical TCA-cycle complex mixture) and succinate (complex II) with or without ADP were used in skeletal muscle.

Results: Preliminary results show a tendency to reduced ROS production in skeletal muscle mitochondria of UCP3Tg mice compared to their Wt-controls both with (peak surface $44.5 \pm 8.5$ vs $32.5 \pm 10.0 ; p=0.10$ ) and without ADP (AUC $22.3 \pm 6.1$ vs $17.2 \pm 1.4, p=0.08$ ). In cardiac muscle mitochondria no difference in ROS production was observed (12.6 \pm 3.1 vs $10.6 \pm 0.5 ; p=0.18)$.

Conclusion: These results suggest that UCP3 is able to directly decrease ROS production in skeletal muscle mitochondria. A reduced UCP3 level in type 2 diabetic patients may therefore contribute to the pathogenesis of type 2 diabetes.

\section{Differential Effects of AMPK $\alpha 2$ Deletion on p70S6K Pathway in Normoxic and Ischemic Hearts and on the Development of Left Ventricular Hypertrophy}

E. Zarrinpashneh ${ }^{1}$, C. Beauloye ${ }^{1}$, A. Ginion ${ }^{1}$, A.-C. Pouleur $^{1}$, X. Havaux ${ }^{1,}$ S. Horman ${ }^{2}$, L.Hue ${ }^{2}$, B. Viollet ${ }^{3}$, J.-L. Vanoverschelde ${ }^{1}$ and L. Bertrand ${ }^{1}$

${ }^{1}$ Division of Cardiology, ${ }^{2}$ Hormone and Metabolic research unit, Université catholique de Louvain, Brussels, Belgium and ${ }^{3}$ Institute Cochin, INSERM, CNRS, René Descartes University, Paris, France.

Background. It has been suggested that AMP-activated protein kinase (AMPK) plays a role in the control of protein synthesis during ischemia. It remains uncertain whether AMPK also participates in the control of protein synthesis under normoxia or during the development of cardiac hypertrophy.

Aim. Accordingly, we sought to evaluate the potential contribution of AMPK to the control of protein synthesis in these different conditions by use of AMPK $\alpha 2$ knock-out $\left(\alpha 2^{-/-}\right)$mice.

Methods. The role of AMPK was tested in isolated hearts perfused with our without insulin and submitted or not to ischemia. The implication of AMPK in the development of cardiac hypertrophy was tested in mice submitted to isoproterenol injections. 
Results. Under normoxia, insulin induced similar activation of protein kinase $\mathrm{B}$ (PKB) and PKB-dependent phosphorylation of mammalian target of rapamycin (mTOR) in both mice. By contrast, the absence of AMPK $\alpha 2$ caused a significant increase of PKBmTOR mediated activation of p70 ribosomal S6 kinase (p70S6K). By contrast, ischemia provoked a similar decrease in insulin-induced PKB/mTOR/ p70S6K stimulation in both WT and $\alpha 2^{-/-}$mice. To determine whether the increase in p70S6K seen in $\alpha 2^{-/-}$influences the development of hypertrophy, mice were submitted to isoproterenol. Echo-derived left ventricular (LV) mass and anterior wall thickness increased more in $\alpha 2^{-/-}$than in WT mice.

Conclusions: Our results show that AMPK $\alpha 2$ plays a role in the regulation of $\mathrm{p} 70 \mathrm{~S} 6 \mathrm{~K}$ activity under normoxia but not under ischemia. Moreover, the absence of this isoform amplifies the isoproterenol-induced LV hypertrophy.

\section{Cardioprotective cGMP Favors Exogenous Fatty Acid Incorporation into Triglycerides over Direct $\beta$-Oxidation}

R. Khairallah', M.E. Young ${ }^{3}$, B.G. Allen ${ }^{2}$, G. Lopaschuk ${ }^{4}$, C. Deschepper ${ }^{2}$, C. Des Rosiers ${ }^{2}$

${ }^{1}$ McGill University, ${ }^{2}$ University of Montreal, ${ }^{3}$ Baylor College of Medicine, ${ }^{4}$ University of Alberta.

While cardiac hypertrophy has been associated with a shift in substrate selection for energy production from fatty acids (FA) to carbohydrates $(\mathrm{CHO})$, it remains controversial whether this shift is adaptive or maladaptive. Since enhanced cGMP signalling can prevent hypertrophy, we hypothesized that this cardioprotection may involve changes in substrate selection. The present study documents the metabolic and functional profiles of hearts from mice overexpressing a constitutively active soluble guanylate cyclase in a cardiomyocyte-specific manner (Tg) using ex vivo perfusion at physiological workload with ${ }^{13} \mathrm{C}$-labeled substrates. Compared to non-Tg littermates, hearts from $\mathrm{Tg}$ mice displayed improved membrane integrity $(38 \pm 14 \%$ lower lactate dehydrogenase release), while sustaining normal cardiac work. These hearts also showed a $38 \pm 9 \%$ lower contribution of exogenous FA to acetyl-CoA formation, while $\mathrm{CHO}$ (both endogenous and exogenous) contribution was similar despite a two-fold increase in glycolytic flux. Since calculated citric acid cycle flux, tissue malonyl-CoA (a $\beta$-oxidation inhibitor), acetyl$\mathrm{CoA}$ and free CoA levels were similar in both groups, these observations suggested an increased contribution from an endogenous FA source, namely triglycerides, in Tg mice hearts. Additionally, we observed a two-fold increase in exogenous oleate incorporation into triglycerides and a decreased (37t $13 \%$ ) phosphorylated AMPK (which inhibits triglyceride hydrolysis) in Tg hearts. Altogether, it appears that cardioprotective cGMP simultaneously may promote both triglyceride hydrolysis and synthesis in cardiomyocytes. Since we detected no differences in the expression of key metabolic genes, the documented changes in substrate selection with chronic activation of cGMP signalling are likely to result from posttranscriptional modifications. (Funded by CIHR)

\section{Uraemic Cardiomyopathy is Characterized by Altered Metabolic Substrate Transport}

Dunja Aksentijević, *Sunil Bhandari, Anne-Marie L. Seymour

Department of Biological Sciences, University of Hull, *Department of Renal Medicine, Hull Royal Infirmary, Hull, UK

Cardiac complications are the leading cause of premature deaths in patients with chronic kidney disease (CKD). Left ventricular hypertrophy (LVH) is a major contributing factor in uraemic cardiomyopathy and results in significant metabolic, cellular and molecular remodelling. Progression of $\mathrm{LVH}$ can lead to the development of insulin resistance, a common feature in CKD and heart failure.

The aim of this study was to investigate the impact of CKD on expression of myocardial glucose and fatty acid transporters (GLUT 4 insulin sensitive transporter, GLUT1 and FAT/CD36 fatty acid transporter).Experimental uraemia was induced surgically via a two-stage 5/6 nephrectomy in adult male Sprague-Dawley rats.

6 weeks later, LV tissue was harvested and the expression of ANF, GLUT 4, GLUT 1 and FAT/CD36 examined using SDS PAGE and Western Blotting.

At this stage, uraemic animals exhibited a significant degree of uraemia (creatinine $69 \pm 2$ vs. $40 \pm 2 \mu \mathrm{mol} / \mathrm{L} n=27 p<0.05$ ), hypertrophy (dry heart weight:tibia length $0.5 \pm 2 \mathrm{vs} .0 .3 \pm 2 \mathrm{~g} / \mathrm{cm}$ $n=23, p<0.05)$, hyperinsulinaemia $(2.1 \pm 0.1$ vs. $1.5 \pm 0.1 \mu \mathrm{g} / \mathrm{L}$, $n=26, p<0.05)$, anaemia ( $29 \pm 1$ vs. $34 \pm 1 \% n=20, p<0.05)$ and hypertension ( $163 \pm 1$ vs. $147 \pm 2 \mathrm{mmHg}, n=25 p<0.05)$.

Uraemic tissue showed a significant increase in myocardial ANF, GLUT 4 and CD 36 expression. (Figure 1, ** $p<0.05$ vs. control) GLUT 1 expression was unaltered.
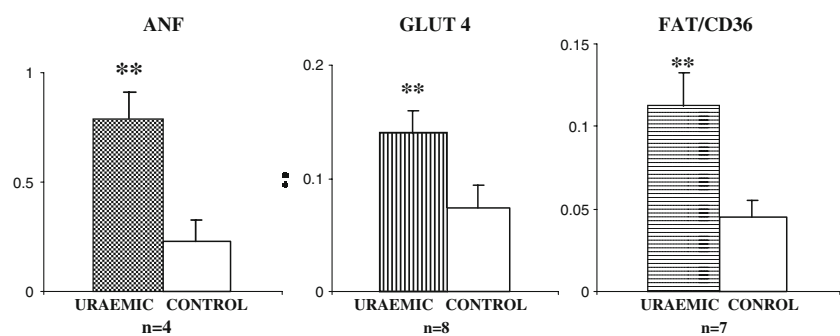

Therefore, 6 week uraemia is characterized by LVH, significant haemodynamic abnormalities and altered ex- 
pression of glucose and fatty acid transporters indicative of developing insulin resistance, remodelling, and ultimately heart failure.

\section{Fatty Acid Transporters in the Hypertrophied Human Heart: Differences Between Atria and Ventricles}

Lisa C Heather ${ }^{\mathrm{a}}$, Neil J Howell ${ }^{\mathrm{b}}$, Mark A Cole ${ }^{\mathrm{a}}$, Michael P Frenneaux $^{\mathrm{c}}$, Domenico Pagano ${ }^{\mathrm{b}}$ and Kieran Clarke ${ }^{\mathrm{a}}$ ${ }^{a}$ Cardiac Metabolism Research Group, Department of Physiology, Anatomy and Genetics, University of Oxford, Oxford, UK. ${ }^{b}$ Department of Cardiothoracic Surgery, University Hospital NHS Foundation Trust, Birmingham, UK. ${ }^{c}$ Department of Cardiovascular Medicine, Birmingham University, $U K$.

Objectives: In the failing human heart, fatty acid metabolism is reduced and correlates with the severity of disease and the degree of hypertrophy. We hypothesized that, in the human heart, the protein levels of the fatty acid transporters, fatty acid translocase (FAT/CD36), cytosolic and plasma membrane fatty acid binding proteins (cFABP and FABPpm) and fatty acid transport proteins (FATP) 1 and 6 , would also vary with the severity of cardiac impairment.

Methods and Results: In biopsies from 20 patients with aortic valve disease, Western blotting revealed significant differences in the protein levels of the fatty acid and glucose transporters between the atrial and ventricular tissue. FAT/ CD36, FATP1, FABPpm, cFABP and GLUT4 were higher in the ventricle, whereas FATP6 was higher in the atria. In the atria, there were positive correlations between FAT/CD36, FABPpm and CFABP, and independently between FATP1 and 6 . In the ventricle, the relationship between FABPpm and cFABP, and between FATP1 and 6 was still present. There was a negative correlation between ventricular FAT/CD36 and GLUT4 protein levels. A correlation between the fatty acid transporters, disease severity or plasma metabolites was not detected.

Conclusions: The inverse relationship between the predominant fatty acid transporter FAT/CD36 and glucose transporter GLUT4 in the ventricle resembles the metabolic shift away from fatty acid oxidation towards glucose utilisation, which occurs in heart failure. In addition, there appears to be different roles, or regulatory mechanisms, for the different fatty acid transporters in human atria and ventricle.

\section{Impact of 3 Week High-Fat Diet on Fatty Acid Metabolism and Gene Expression in Cardiac Hypertrophy}

Ashwin Akki and Anne-Marie L. Seymour, Department of Biological Sciences, University of Hull, Hull $U K$.
Impaired fatty acid (FA) oxidation in the setting of cardiac hypertrophy $(\mathrm{CH})$ can lead to inappropriate accumulation of lipids, which may underlie deterioration into heart failure. We investigated alterations in FA metabolism in $\mathrm{CH}$ in response to high-fat feeding. $\mathrm{CH}$ was induced in male Sprague-Dawley rats by abdominal aortic constriction (AC). Group 1 received normal diet for 9 weeks postsurgery whilst Group 2 received a high-fat diet $(45 \% \mathrm{kcal}$ from lipids) from weeks 6 to 9 . Substrate oxidation was determined in perfused hearts using ${ }^{13} \mathrm{C}$ NMR and gene expression probed using real-time PCR. In Group-1, myocardial expression of PPAR $\alpha$ and PPAR $\alpha$-regulated genes was significantly down-regulated in AC vs. Control (Con), consistent with a marked reduction in palmitate oxidation. In Group-2, expression of PPAR $\alpha, \mathrm{mCPT} 1$ and MCAD was markedly upregulated in AC with normalisation of palmitate oxidation [AC $37 \pm 3 \%$ vs. Con $36 \pm 4 \%$ ] whilst UCP3 expression remained low. Function declined markedly [Rate pressure product $\times 10^{3}(\mathrm{mmHg} / \mathrm{min}) \mathrm{AC} 19 \pm$ $1 \dagger$ vs. Con $18 \pm 1 \#, \uparrow p<0.05$ vs. AC in Group- $1, \# p<0.05$ vs. Con in Group-1].

\begin{tabular}{|c|c|c|c|c|c|}
\hline & Mode & $\begin{array}{l}1 \text { PPAR } \alpha \\
\text { (Peroxisome } \\
\text { proliferator- } \\
\text { activated } \\
\text { receptor } \alpha \text { ) }\end{array}$ & $\begin{array}{l}\text { mCPT1 } \\
\text { (Carnitine } \\
\text { palmitoyl } \\
\text { transferase) }\end{array}$ & $\begin{array}{l}\text { MCAD } \\
\text { (Medium- } \\
\text { chain } \\
\text { acylCoA } \\
\text { dehydrogenase) }\end{array}$ & $\begin{array}{l}\text { UCP3 } \\
\text { (Uncoupling } \\
\text { protein) }\end{array}$ \\
\hline \multirow[t]{2}{*}{$\begin{array}{c}\text { Group 1 } \\
(n=4)\end{array}$} & Con & 1.0 & 1.0 & 1.0 & 1.0 \\
\hline & $\mathrm{AC}$ & $0.4 \pm 0.1 *$ & $0.5 \pm 0.1^{*}$ & $0.7 \pm 0.0^{*}$ & $0.4 \pm 0.1 *$ \\
\hline \multirow[t]{2}{*}{$\begin{array}{c}\text { Group } 2 \\
(n=4)\end{array}$} & Con & $5.1 \pm 0.6^{*}$ & $2.6 \pm 0.3^{*}$ & $1.6 \pm 0.1^{*}$ & $1.3 \pm 0.2$ \\
\hline & $\mathrm{AC}$ & $2.7 \pm 0.2 *$ & $1.5 \pm 0.1 *$ & $1.4 \pm 0.1 *$ & $0.5 \pm 0.1 *$ \\
\hline
\end{tabular}

Data normalised to Calsequestrin-2 in each group. ${ }^{*} p<0.05$ vs. Con in Group 1

Three weeks of high-fat feeding reversed metabolic and transcript adaptations in $\mathrm{CH}$ but with a marked deterioration in function. In conclusion, high-fat feeding exacerbates contractile dysfunction in $\mathrm{CH}$ despite a reversal of cellular remodelling.

Erythropoietin Treatment Normalises Fatty Acid Oxidation, but not Function, in the Chronically Infarcted Rat Heart

Mark Cole ${ }^{1}$, Carolyn Carr ${ }^{1}$, Lisa Heather ${ }^{1}$, Emma Carter $^{1}$, Regien Schoemaker ${ }^{2}$, Kieran Clarke ${ }^{1}$

${ }^{I}$ Dept. of Physiology, Anatomy and Genetics, University of Oxford, Parks Rd, Oxford, UK ${ }^{2}$ Dept. of Experimental Cardiology, University Medical Centre, Groningen, $N L$ 
In the chronically infarcted rat heart, oxidation of fatty acids is reduced, as are other markers of cardiomyocyte fatty acid handling (Heather et al, 2006). In vivo function in the infarcted rat heart has been improved using erythropoietin (EPO) treatment (Van de Meer et al. 2005), suggesting that oxygen delivery is important after infarction and may underlie changes in fatty acid metabolism. Here, we examined the effect of EPO treatment on oxygen consumption and fatty acid oxidation in the chronically infarcted rat heart. Myocardial infarction was performed in male Wistar rats $(200 \mathrm{~g}, n=15)$. A subset of 8 infarcted animals was subsequently treated with $40 \mu \mathrm{g} / \mathrm{kg}$ EPO i.p. at 3 and 6 weeks post infarction (EPO infarcts). Infarcted hearts at 10 weeks had ejection fractions of $45 \pm 4 \%$ compared with $74 \pm 3 \%$ in sham-operated animals $(n=8, p<0.01)$, assessed using in vivo cine MRI. EPO treatment did not improve the ejection fraction. The heart/body weight ratios of all infarcted rats were the same with or without EPO treatment, and were significantly greater than sham-operated rats. At 15 weeks post infarct, hearts were excised and perfused in Langendorff mode with Krebs-Henseleit buffer containing $11 \mathrm{mM}$ glucose plus $0.4 \mathrm{mM}$ palmitate, containing $0.2 \mu \mathrm{Ci} \mathrm{ml}{ }^{-1}$ $\left[9,10-{ }^{3} \mathrm{H}\right]$ palmitate. Rate pressure products for all infarcted rat hearts were significantly lower than shams, and correlated positively $(p<0.001)$ with the in vivo ejection fractions (see below). Oxygen consumption, measured using chemiluminescence, was reduced to the same extent in all infarcted rat hearts compared with shams $(p<0.05)$. However, fatty acid oxidation was significantly reduced in infarcted rat hearts compared to shams, but not in EPO-treated infarcted hearts. EPO treatment therefore prevented the decrease in fatty acid oxidation associated with chronic myocardial infarction. Functional improvement may result from more prolonged EPO treatment.

\section{References}

Heather et al. Cardiovasc Res. 72: 430-7, 2006.

Van de Meer et al. J Am Coll Cardiol. 46: 1 25-33, 2005.
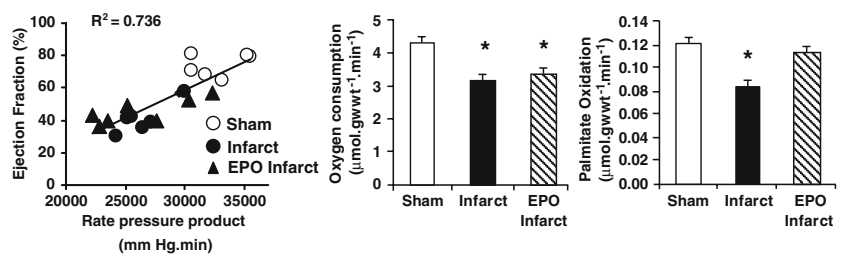

Opposite Correlations of Cardiac Palmitate Oxidation Rates and Plasma Free Fatty Acids with Ejection Fractions in the Infarcted Rat Heart

Anthony F Catchpole, Carolyn A Carr, Lisa C Heather, Kieran Clarke

Department of Physiology, Anatomy and Genetics, University of Oxford, Oxford, UK

Background: Under normal physiological conditions the heart derives most of its energy from fatty acid oxidation. Infarcted, failing rat hearts six months after surgery have decreased palmitate oxidation rates.

Objectives: To determine the degree of cardiac impairment in rat hearts ten weeks after infarction, whether cardiac palmitate oxidation rates are increased or decreased, and whether the two are related.

Methods: Male Wistar rats were subjected to left coronary artery ligation $(n=5)$ or sham operation $(n=6)$. Cardiac function and morphology were evaluated using cine MRI at one and ten weeks. At 10 weeks, ${ }^{3} \mathrm{H}$-palmitate oxidation was determined in isolated, Langendorff-perfused hearts. Medium chain acyl-CoA dehydrogenase (MCAD) activity and fasting plasma free fatty acid (FFA) concentration were determined using enzyme assays.

Results: At one week, infarcted heart end diastolic volumes were increased by $29 \%(p<0.05)$. At ten weeks end diastolic volumes were increased by $55 \%(p<0.001)$ and left ventricular mass increased by $11 \%(p<0.01)$, compared with shams. At ten weeks, cardiac palmitate oxidation correlated positively, and plasma FFA concentration correlated negatively, with ejection fractions (see figure). Left and right ventricular MCAD activity and lung wet weight/dry weight ratio were the same for both groups.

Conclusions: Rat hearts were hypertrophied and dilated at ten weeks after infarction, but pulmonary congestion and reduced MCAD levels, suggesting heart failure, were not observed. Despite the elevated plasma FFA levels in infarcted animals, rates of cardiac palmitate oxidation were decreased, potentially caused by reduced cardiomyocyte FFA uptake.
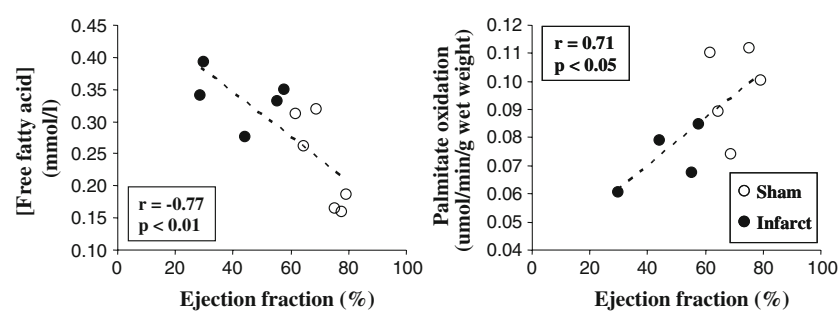


\section{Skeletal Muscle Function in Aortic Banding Cardiac Hypertrophy}

Renee Henry ${ }^{1}$, Peter L McLennan ${ }^{2}$, Gregory E Peoples ${ }^{1}$. School of Health Sciences ${ }^{l}$ and Graduate School of Medicine ${ }^{2}$, University of Wollongong, Australia

Background: Skeletal muscle fatigue and dysfunction has been reported in rats using a myocardial infarction model of heart failure, however there is very little evidence regarding skeletal muscle dysfunction in the aortic banding model of cardiac hypertrophy.

Objectives: This project aimed to determine whether skeletal muscle dysfunction is present in rats with aortic banding-induced pressure overload.

Methods: Male Sprague-Dawley rats (9-10w) underwent abdominal aortic banding or sham surgery. Muscle function tests were performed $15 \mathrm{w}$ following surgery using the auto-perfused hind limb. Contractile properties of the gastrocnemius-soleus-plantaris (GSP) muscle bundle were determined using two protocols: repetitive single twitch contractions $(1 \mathrm{~Hz}, 5 \mathrm{~min})$ with $50 \mathrm{~Hz}, 1 \mathrm{sec}$ tetanus before and after and; repeated bouts of low-frequency stimulation ( $5 \mathrm{~Hz}$ for $5 \mathrm{~s}$ of every $10 \mathrm{~s}$, for $5 \mathrm{~min}$ ).

Results: Aortic banding resulted in a $22 \%$ increase in heart weight to tibia length ratio after $15 \mathrm{w}$ (mean \pm SEM sham vs banded, $2.85 \pm 0.06$ vs $3.47 \pm 0.26, p<0.01)$. Lung weight to tibia length ratio was not different in banded rats $(2.76 \pm$ 0.09 vs $2.77 \pm 0.13, p=0.93$ ) nor was muscle size (GSP to tibia length ratio, $6.77 \pm 0.21$ vs $6.65 \pm 0.32, p=0.75$ ). There was no effect of banding on any parameter of muscle contractile function analysed (absolute forces and contraction and relaxation rates, rates of fatigue; all $\mathrm{p}>0.05$ ) in either stimulation protocol.

Conclusions: Although there was significant cardiac hypertrophy observed with banding there were no symptoms of heart failure, indicating that after 15 weeks the heart was in a state of compensated hypertrophy. In this compensated state, there was no early indication of skeletal muscle dysfunction.

\section{Regulation of Myocardial Metabolic Gene Expression in Pressure-Overload and Exercise-induced Hypertrophy}

Michael Schwarzer, Christian Riehle, Gracjan Pytel, Heiko Bugger, Daniel Blum, Torsten Doenst

University of Leipzig and Heart Center Leipzig, Leipzig, Germany
Background: Hypertrophy can be induced by pressureoverload or treadmill running. Pressure-overload hypertrophy has been associated with distinct changes in gene expression. The regulatory mechanisms in exercise-induced hypertrophy are largely unknown.

Objective: To compare the expression of metabolic genes and their regulators in exercise-induced and pressureoverload hypertrophy.

Methods: Exercise-induced hypertrophy was induced by treadmill running of male Sprague Dawley rats for 10 weeks. Pressure-overload hypertrophy was induced by aortic banding for 14 days. Contractile function was assessed by echocardiography. Expression of glucose (GO) and fatty acid oxidation (FAO) genes, as well as regulators of metabolic gene expression, were assessed by RT-PCR.

Results: Both interventions caused significant hypertrophy (heart-to-body-weight ratio $+17.0 \%$ Banding, $+14.3 \%$ Exercise, $p<0.05$ ) without affecting left ventricular function (normal EF). Contrary to expectations, exercise-induced hypertrophy was not associated with any changes in the expression of FAO genes or the PPARg-coactivators (PGC)-1a and $-1 b$. In contrast, pressure-overload showed a clear trend towards reduced expression of FAO-genes (MCAD $-43 \%$ $\mathrm{p}<0.05$, LCAD $-20 \%, p<0.05$, FABP $-26 \%, p<0.01$, CPT1 $-20 \%$, FATP $-19 \%$ ), accompanied by reduced expression of PGC-1a and $-1 \mathrm{~b}(-40 \%$ and $-35 \%$, respectively, $\mathrm{p}<0.01)$. Expression of putative suppressors of PGC-1a was either unchanged or even reduced both in exercise-induced and pressure-overload hypertrophy (Akt, H11K, Cdk9, HIF1a).

Conclusions: Pressure-overload and exercise-induced hypertrophy appear to have different regulatory mechanisms governing substrate selection and oxidation. The lack of major differences in expression patterns after treadmill running may be a reflection of a new steady state of gene expression as part of a physiological hypertrophic response.

\section{Development of Hypertrophy and Heart Failure by Minimally Invasive Aortic Constriction in C57/B16 Mice}

Gloria Färber, H. Frederico Barreto-Pereira, Ralf Gilsbach, Michael Schwarzer, Lutz Hein, Torsten Doenst University of Leipzig and Heart Center Leipzig, Germany

Objective: To assess the ability of minimally invasive transverse aortic constriction to induce heart failure in wildtype mice and assess the role of PGC- $1 \alpha$ and $\beta$ and their regulatory targets. 
Methods: Transverse aortic constriction (TAC) was performed through a mini-sternotomy without intubation. The aortic arch was constricted to $27 \mathrm{G}(n=40$, sham $n=12)$. Hypertrophy was assessed by the heart/body-weight ratio and histological analyses. Contractile function was assessed by intracardiac pressure measurements using a Millar catheter. Selected metabolic genes were assessed with quantitative RT-PCR.

Results: Perioperative survival averaged at $70 \%$. Two months after the operation, TAC induced significant hypertrophy (ventricle-body weight ratio: TAC $7.3 \pm 0.5 \mathrm{mg} / \mathrm{g}$ vs. sham $4.5 \pm 0.1 \mathrm{mg} / \mathrm{g}, \mathrm{p}<0.05) .25 \%$ of the TAC mice displayed signs of heart failure (pleural effusions, dyspnea, weight loss). All of them had ventricle-to-body-weight ratios above 10. TAC mice with ventricle-to-body-weight ratios between 6 and 10 demonstrated compensated hypertrophy with normal $\mathrm{d} p / \mathrm{d} t_{\max }$ $(6621 \pm 774 \mathrm{mmHg} / \mathrm{sec}$ vs. sham $6606 \pm 569 \mathrm{mmHg} / \mathrm{sec})$ and no clinical heart failure signs. Rate-pressure products $\left(\mathrm{min}^{-1} \mathrm{x} \mathrm{mmHg} / \mathrm{mgBW}\right)$ were $508 \pm 38$ in sham, $446 \pm 30$ in hypertrophied (n.s.), and $157 \pm 64$ in failing hearts $(\mathrm{p}<0.01)$. Both hypertrophied and failing hearts showed increased amounts of fibrosis. There was no change in PGC-1a and -b expression in hypertrophied heart. In heart-failure, PGC-1b was near normal but PGC- $1 \alpha$ was down-regulated to $32 \pm$ $7.6 \%$ of control $(p<0.01$, consistent with decreased PPARg and fatty acid oxidation gene expression.

Conclusions: Minimally invasive transverse aortic constriction causes heart failure in a significant fraction of wildtype mice. This pressure-induced heart failure is indeed associated with a down-regulation of PGC- $1 \alpha$ and $\beta$ oxidation genes.

\section{Autophagy Regulates Remodeling in Unloaded Heart}

Mitra Rajabi, Rebecca L Salazar, Peter Razeghi, Stanislaw Stepkowski, Heinrich Taegtmeyer,

University of Texas-Houston, Houston, TX, USA

Background-Clinical reports have shown that left ventricular assist device implantation may improve cardiac function in patients with end-stage heart failure. This has been associated with a decrease in cardiomyocyte size. The mechanisms for unloading-induced cardiomyocyte atrophy (reverse remodeling) are not fully understood. In failing heart apoptotic and autophagic cell death are increased. Whether autophagy changes with mechanical unloading is unknown.

Hypothesis-Autophagy is activated in mechanical unloading of the failing heart.

Methods-Male rats underwent heterotopic heart transplantation (transplantation of a rat heart into a heterotopic position). Animals were sacrificed at different time points after the surgery, then the native and the transplanted hearts were removed, freeze clamped, weighed, and processed for protein extraction and histology. The heart to body weight ratio was calculated and compared between groups as an indicator of atrophy. To determine autophagic activity we measured expression of microtubule-associated protein light chain 3 (LC3), which is a specific marker to monitor autophagy.

Results-As expected, the heart weight to body weight ratio of the unloaded hearts decreased by $24 \%$ and $56 \%$, one week and one month after transplant, respectively. The histology revealed a significant decrease in ventricular cardiomyocytes size in unloaded hearts. Protein analysis using anti LC3 antibody showed increased expression of LC3 II in unloaded hearts demonstrating ongoing autophagic activity in those samples.

Conclusion-Mechanical unloading of the heterotopic transplanted rat heart shows activation of autophagy which may play a role in atrophic remodeling of heart through degradation of proteins and unused organelles after unloading.

\section{Calpain 1 Gene Expression and Calpain-Like Protease Activity Increase with Atrophic Remodeling of the Heart}

Peter Razeghi, Kedryn Baskin, Kaelin Volpini, Heinrich Taegtmeyer

Department of Internal Medicine, Division of Cardiology, University of Texas Health Science Center at Houston TX, USA

Background: Cardiac hypertrophy is an independent risk factor for the development of heart failure. Regulators of cardiomyocyte atrophy are potential targets for reversing cardiac hypertrophy. Calpain 1 and calpain 2 are essential regulators of skeletal muscle atrophy. Their role in heart muscle is undefined.

Hypothesis: Calpain 1 and 2 regulate cardiomyocyte atrophy.

Methods: We used two models of unloading-induced cardiac atrophy and one model of starvation-induced cardiomyocyte atrophy. Failing human hearts of 10 patients ( 8 men, age: $40.4 \pm 5.7)$ were unloaded with a left ventricular assist device (mean duration was $200 \pm 38$ days, range: 64-437 days). Myocardial samples were obtained from failing human hearts before and after mechanical unloading. Normal rat hearts were unloaded for 7 days by heterotopic transplantation into the abdomen of a recipient rat. Neonatal rat ventricular cardiomyocytes (NRVM) were starved of amino acids for 18 hours. Using quantitative RTPCR we measured transcript levels of calpain 1 and calpain 
2 at baseline and after treatment. In the unloaded rat hearts we also measured calpain-like protease activity.

Results: In patients with left ventricular systolic dysfunction, mechanical unloading increased cardiac transcript levels of calpain 1 but unloading did not significantly change calpain 2 gene expression. The change in calpain 1 transcript levels correlated negatively with the duration of unloading $\left(r^{2}=-0.73, p<0.01\right)$. Unloading of the heterotopically transplanted rat heart significantly increased calpain 1 transcript levels and calpain-like protease activity. In nutrient deprived NRVM (no amino acids) calpain 1 transcript levels increased.

Conclusions: Calpain 1 gene expression and activity increases during cardiomyocyte atrophy suggesting that calpain 1 may regulate reversal of hypertrophy. Future studies will examine the trophic effect of calpain 1 inhibitors in models of atrophy and calpain 1 activators in models of hypertrophy.

\section{Excess Endothelin-1 and Reperfusion Injury in Rabbit Myocardial Infarction and Isolated Rabbit Cardiomyocytes}

Sophie Tamareille*, James Amirian*, Patricia Felli*, Yong J. Geng*, Fenghua Li ${ }^{\dagger}$, William H. Barry ${ }^{\dagger}$, Richard W. Smalling*

Division of Cardiovascular Medicine, University of Texas at Houston Medical School* and University of Utah Health Science Center, Salt Lake City ${ }^{\dagger}$

Background-The mechanisms by which left ventricular (LV) unloading limits infarct size in acute myocardial infarction are unknown.

Hypothesis - We tested the hypothesis that endothelin-1 (ET-1) causes myocyte calcium $\left(\mathrm{Ca}^{2+}\right)$ overload which results in myocardial reperfusion injury and can be inhibited by LV unloading immediately prior to reperfusion. Methods - 12 anesthetized rabbits were subjected to 1 hour of left circumflex coronary artery occlusion followed by 3 hours of reperfusion. Unloading was initiated $15 \mathrm{~min}$ prior to reperfusion and maintained during the entire period of reperfusion (unloaded group, $n=6)$. A control group $(n=6)$ was subjected to reperfusion alone. In parallel, isolated rabbit myocytes were subjected to simulated ischemia/ reperfusion with and without ET-1 and intracellular $\mathrm{Ca}^{2+}$ was assessed with flow cytometry.

Results_-Just prior to reperfusion, LV unloading significantly decreased the LV end-diastolic pressure. Infarct size, expressed as a percentage of zone at risk, was also significantly reduced in the unloaded group compared to controls $(3.39 \pm 1.35 \%$ vs $17.50 \pm 4.52 \%, P=0.013)$. Unloading support significantly reduced ET-1 release from the heart at 2 hours of reperfusion $(21.37 \pm 2.83 \mathrm{pg} / \mathrm{mL}$ vs $54.34 \pm$ $11.34 \mathrm{pg} / \mathrm{mL}, P=0.03)$. LV unloading caused a significant reduction in the $\%$ of apoptotic cells $(2.75 \pm 0.38 \%$ vs $5.84 \pm$ $1.97 \%, P=0.003)$ in the ischemic region. In isolated ventricular myocytes subjected to simulated ischemia/ reperfusion, ET-1 at physiologic levels increased $\mathrm{Ca}^{2+}$ uptake and decreased myocyte viability.

Conclusions-Our results suggests that ET-1 release by ischemic myocardium at the time of reperfusion results in fatal myocardial $\mathrm{Ca}^{2+}$ overload and is likely an important mediator of reperfusion injury.

\section{Malonyl CoA Decarboxylase Deficient Mice Display Minimal Infarct During In Vivo Ischemia/Reperfusion}

Wei Wang, John Edward R. Ussher, Shaohua Wang, Jason R.B. Dyck, Gary D. Lopaschuk

Cardiovascular Research Group. Departments of Pediatrics, Pharmacology, and Surgery, University of Alberta, Canada

During reperfusion of the ischemic myocardium, fatty acid oxidation (FAO) rates quickly recover, while glucose oxidation (GOX) rates remain low. This results in an uncoupling between glycolysis and glucose oxidation, leading to an increased proton production, which decreases cardiac efficiency during the critical stages of reperfusion. Malonyl CoA decarboxylase (MCD), an enzyme responsible for degrading malonyl CoA, relieves malonyl CoAdependent inhibition of carnitine palmitoyl transferase-1, resulting in an increased rate of FAO. Studies have shown that inhibition of MCD improves the reperfusion recovery of ischemic hearts by reducing rates of FAO and increasing rates of GOX. The objective of this study was to determine whether inhibition of MCD results in decreased infarct size. WT or MCD knockout $\left(\mathrm{MCD}^{-/}\right)$mice were anaesthetized and subjected to temporary left anterior descending coronary artery ligation for $30 \mathrm{~min}$ followed by $24 \mathrm{hrs}$ of reperfusion. At the end of reperfusion, hearts were removed and perfused with Evans blue to delineate the area at risk, and then stained in $1 \%$ triphenyltetrazolium chloride at $37^{\circ} \mathrm{C}$ for $10 \mathrm{~min}$ to determine infarct size. $\mathrm{MCD}^{-/-}$mice demonstrated a dramatic reduction in overall infarct size compared to WT mice $(10.80+4.04 \%$ vs. $39.50+4.01 \%$ of area at risk) with similar areas at risk $(53.00+8.99 \%$ vs. $51.90+5.93 \%$ of left ventricle).

This study demonstrates that inhibition of MCD may be beneficial for the treatment of ischemia/reperfusion injury, further validating the optimization of cardiac energy metabolism as a novel therapy for ischemic heart disease. 
AMPK and Fatty Acids Synergistically Induce Pdk4 Expression to Reduce Glucose Oxidation

Malika Chegary, Heleen te Brinke, Frits Wijburg, Ronald Wanders, Sander Houten

Laboratory Genetic Metabolic Diseases, Departments of Clinical Chemistry and Pediatrics, Academic Medical Center, Amsterdam, the Netherlands.

Background: Heart disease is a common symptom in children with inherited mitochondrial long-chain fatty acid b-oxidation (FAO) defects. It is unknown whether this is caused by an energy shortage due to the FAO defect and/or an accumulation of toxic FAO intermediates. In theory glucose oxidation can compensate for a defect in FAO. Glucose oxidation, however, is decreased by fatty acids, a process in which pyruvate dehydrogenase kinase 4 (PDK4) plays an important role. Elevated PDK4 levels have been reported in a FAO-deficient mouse model.

Hypothesis/objectives: To determine the effect of a FAO defect on PDK4 expression levels and glucose metabolism. Methods: We studied the transcriptional regulation of PDK4 in so-called "Fao" cells. "Fao" cells are characterized by substantial PDK4 expression levels and no lactate production. FAO was inhibited using L-aminocarnitine. Glucose oxidation and FAO were assessed using radiolabeled substrates.

Results: We confirm that PDK4 expression is induced by fatty acids. Inhibition of FAO by L-aminocarnitine did not have any effect on PDK4 expression. In contrast, fatty acids and AMP-activated protein kinase (AMPK) activation by AICAR synergistically induced PDK4 expression. During hypoxia, which also activates AMPK, fatty acids induced PDK4 expression more effectively than during normoxia. AMPK activation decreased glucose oxidation and as a consequence increased lactate formation, producing a decreased nucleotide triphosphate pool.

Conclusions: AMPK and fatty acids synergistically increase PDK4 expression and decrease glucose oxidation. This cooperation makes cells more dependent on FAO. In the case of an inherited FAO defect this is a maladaptation.

\section{Impact of Hormone Replacement Therapy on Myocardial Fatty Acid Metabolism}

Pablo F. Soto, Pilar Herrero, Tingting Turski, Ken Schechtman, Valerie Ratts and Robert J. Gropler.

Washington University School of Medicine, St. Louis, MO.

Background: Studies in experimental animals demonstrate that estrogen (E) increases myocardial fatty acid oxidation (MFAO). Recently, we reported that myocardial oxygen consumption $\left(\mathrm{MVO}_{2}\right)$ as well as MFAO were increased in post-menopausal women receiving $\mathrm{E}$, and that progesterone appeared to attenuate this effect. However, the study was retrospective.

Hypothesis/ Objectives. To determine, prospectively, if short-term administration of $\mathrm{E}$, alone or in combination with progesterone (EP), will alter $\mathrm{MVO}_{2}$ or MFAO in postmenopausal women.

Methods. Fifteen post-menopausal women (age $63 \pm 7$ yrs) not previously on hormone replacement therapy (HRT) underwent quantitative assessment of myocardial blood flow (MBF), $\mathrm{MVO}_{2}$, and fractional fatty acid uptake (MFAuptake) as well as overall fatty acid utilization (MFAU) and MFAO using positron emission tomography with ${ }^{15} \mathrm{O}$-water, ${ }^{11} \mathrm{C}$-acetate and ${ }^{11} \mathrm{C}$-palmitate, respectively. Rate pressure product (RPP) was used to estimate cardiac work. Measurements were performed at baseline and after 3 -days of estradiol ( $0.3 \mathrm{mg} /$ day via transdermal patch) and either progesterone (200 mg/day orally) or placebo.

Results: After $\mathrm{E}$ or $\mathrm{EP}$, both $\mathrm{MBF}$ and $\mathrm{MVO}_{2}$ were unchanged from baseline. Additional findings:

\begin{tabular}{|c|c|c|c|c|c|c|}
\hline \multirow{2}{*}{ Means \pm SD } & \multicolumn{3}{|c|}{$\mathrm{E}(n=7)$} & \multicolumn{3}{|c|}{$\mathrm{EP}(n=8)$} \\
\hline & Baseline & $(+) \mathrm{E}$ & $p$ & Baseline & $(+) \mathrm{E}+\mathrm{P}$ & $P$ \\
\hline Free Fatty Acid & $655 \pm$ & $502 \pm$ & .03 & $686 \pm$ & $851 \pm$ & .11 \\
\hline$(\mathrm{nmol} / \mathrm{mL})$ & 332 & 188 & & 207 & 313 & \\
\hline RPP & $7751 \pm$ & $7224 \pm$ & .01 & $7338 \pm$ & $7044 \pm$ & NS \\
\hline$\left(\mathrm{mm} \mathrm{Hg} \min ^{-1}\right)$ & 1503 & 1782 & & 2852 & 1929 & \\
\hline $\begin{array}{l}\text { MFA uptake } \\
\left(\mathrm{mL} \mathrm{g}^{-1} \mathrm{~min}^{-1}\right)\end{array}$ & $.34 \pm .08$ & $.36 \pm .05$ & NS & $.32 \pm .06$ & $.34 \pm .03$ & NS \\
\hline $\begin{array}{l}\text { MFAU } \\
\qquad\left(\mathrm{nmol} \mathrm{g}^{-1} \mathrm{~min}^{-1}\right)\end{array}$ & $143 \pm 93$ & $105 \pm 36$ & .17 & $121 \pm 44$ & $167 \pm 75$ & .07 \\
\hline $\begin{array}{l}\text { MFAO }\left(\mathrm{nmol} \mathrm{g}^{-1}\right. \\
\left.\min ^{-1}\right)\end{array}$ & $133 \pm 89$ & $95 \pm 35$ & .18 & $106 \pm 47$ & $143 \pm 63$ & .02 \\
\hline MFAU/RPP $\times 10^{4}$ & $19 \pm 13$ & $15 \pm 6$ & NS & $18 \pm 8$ & $24 \pm 12$ & .07 \\
\hline $\mathrm{MFAO} / \mathrm{RPP} \times 10^{4}$ & $18 \pm 13$ & $14 \pm 6$ & NS & $13 \pm 5$ & $20 \pm 9$ & .01 \\
\hline
\end{tabular}

Conclusions: Short-term E therapy lowers plasma FFA and tends to lower overall MFAU or MFAO independent of cardiac work. Combined EP administration increases MFAU and MFAO relative to the amount of cardiac work performed. These changes appear to be mediated by peripheral changes in plasma FFA. The long-term effects of HRT on cardiac metabolism and its impact on cardiac risk profile and function remains to be determined.

Open Access This article is distributed under the terms of the Creative Commons Attribution Noncommercial License which permits any noncommercial use, distribution, and reproduction in any medium, provided the original author(s) and source are credited. 